\title{
TOPOGRAPHIC SPACES OVER ORDERED MONOIDS
}

\author{
JAN PAVLÍK
}

\begin{abstract}
A topography on a set is considered to be a collection of features described by two valuations: distance and elevation. Spaces with such structure will be studied on a general level, with generalized metric (gem) and pseudometric (pseudogem). We show many pseudogem distances and characteristics, particularly those related to the elevation function. We focus on paths in the topographic images and cohesiveness (generalized continuity) of their compositions with the elevation function. Special emphasis is also placed on the spaces arising from the digital geometry and, therefore, offering applications in image processing.
\end{abstract}

\section{INTRODUCTION}

Topography, by one of its meanings, is a tool for describing the placement of various objects in a region. To formalize this vague concept, we may regard each object as a set of points of a given property, thus one can ask where in the region which property is satisfied. This can be seen easily when considering the topography of a landscape, where the considered property is the elevation of a given point. Thus, the topography is determined fully by the elevation function defined on the region. We take this example as a model of a topographic space for further generalization.

We formalize the definition using this approach, which covers many situations in mathematics, namely geometry, and its applications in geography, physics, economy and other fields. The common feature of all these applications is a basic space - a set of elements with a given measure of dissimilarity, i.e., a generalized distance, and a mapping which assigns to each element a value from an ordered set. The dissimilarity may differ from the usual metric by both the defining properties and the set of values - a value algebra. Particularly, the latter case will be subject of our study.

Whenever topographic spaces are defined, we show some of their properties in terms of generalized metric spaces and the induced topology. Finally, we focus on instances of the topographic spaces in the digital geometry and show how some general properties can be helpful for the treatment of digital images.

The foundations for this research are given in [15] where spaces with generalized metric (gem) are studied. Although we will refer to it, we still present, in the first

$M S C$ (2010): primary 46A19; secondary $68 \mathrm{U} 10$.

Keywords: generalized metric space; ordered monoid; path connectedness; topography.

The author acknowledges the support by the project CZ.1.07/2.3.00/30.0005 of Brno University of Technology. 
section, all necessary definitions and propositions proven there. In contrast to that paper, we introduce the concept of c-cohesiveness which generalizes that of cohesiveness, a modification of continuity. As in metric spaces, each pair of elements is assigned a path-distance, i.e., infimum of the lengths of paths connecting the points. This assignment induces a new gem and we are interested in its abstract version, which involves free meet quantale over a monoid. It is shown that we can get a family of new gems if we have suitable assignments on paths in the space. In the second section, we introduce the comcept of a topographic space with some of its connectedness properties shown. The third section contains the most results, a majority of them dealing with path characteristics in the topographic space, namely those related to elevation gain and prominence which are notions used in public. One of the main results is Theorem 4.15, which clarifies the relationship between the passability of the spaces and connectedness of the space with respect to prominence distance. This might be helpful for the detection of (near) passability of the space. Some examples are shown and applications are sketched in the last section.

Throughout this paper let $\underline{\mathbb{N}}=\mathbb{N} \cup\{0\}$ and $\underline{\mathbb{R}}=\{x \in \mathbb{R} \mid x \geq 0\}$ be the set of non-negative reals.

\section{Generalized metric spaces}

In order to define a generalized metric space, the fundamental structure for our research, we need to clarify which algebraic structures - value monoids - we will use as target sets for distances.

\subsection{Value monoids and generalized distances}

Throughout this framework we work with ordered commutative monoids, i.e., commutative monoids where the addition is isotone in both components and 0 is the least element. Now we recall the definitions of generalized metric and related concepts $[3,5,14,15,18]$.

Definition 2.1. Given an ordered commutative monoid $(M,+, 0, \leq)$ and a set $X$, a mapping $\delta: X^{2} \rightarrow M$ satisfying

- $\delta(x, y)=0 \Leftrightarrow x=y$ (strong self-uniformity),

- $\delta(x, y)=\delta(y, x)$ (symmetry),

- $\delta(x, y)+\delta(y, z) \geq \delta(x, z)(\triangle$-inequality $)$

is called a generalized metric (or gem) and $(X, M, \delta)$ is referred to as a gem space. Elements of gem spaces are mostly called points. Weaker versions of generalized distances are used as well. If the first property is satisfied only as a " $\Leftarrow$ " implication, then we get a generalized pseudometric (abbreviated as a pseudogem). If the second and the third, respectively, property is violated, then we get a generalized quasimetric and dissimilarity, respectively.

If $M$ is a complete lattice and + is a join operation, then gem $\omega$ becomes a generalized ultrametric (or gum). Recall, that an ultrametric $\delta$ on $\mathbb{R}^{n}$ is a metric whose triangle inequality is satisfied in a stronger form

$$
\max \{\delta(x, y), \delta(y, z)\} \geq \delta(x, z) .
$$


Generalized ultrametric spaces in the general setting are closely connected with the theory of lattice-valued fuzzy sets and have been studied in the context of digital spaces, see $[1,6,9,14]$. A digital space is a connected undirected graph whose edges are given in terms of reflexive symmetric binary relation called adjacency.

Now we recall some well known and obvious facts on generalized metric spaces.

Proposition 2.2. (1) Every mapping to a pseudogem space induces a pseudogem on the original space. Indeed, for a pseudogem space $(X, M, \delta)$ and a mapping $f: Y \rightarrow X$ we have a pseudogem $\delta_{f}$ on $Y$ with values in $M$ given by $\delta_{f}(x, y)=\delta(f(x), f(y))$.

(2) A collection of pseudogems on a given set gives rise to a pseudogem (a gem, if the collection includes a gem) with values in the product of the value algebras. Namely, given a set $\left\{\left(X, M_{i}, \delta_{i}\right) \mid i \in I\right\}$ of pseudogem spaces, then there is a mapping $\left(\delta_{i}\right)_{i \in I}: X \rightarrow \prod_{i \in I} M_{i}$ which factorizes over the submonoid $M$ of $\prod_{i \in I} M_{i}$ generated by the set $\underline{M}$ of values of $\left(\delta_{i}\right)_{i \in I}$.

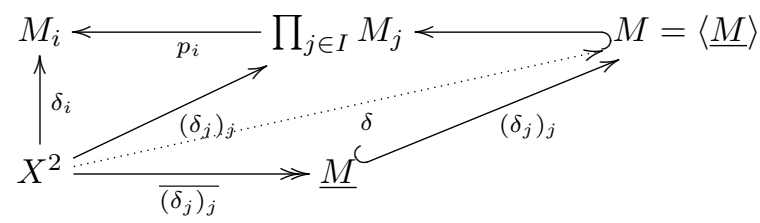

The unique factorization is then a pseudogem and if there exists some $i_{0} \in I$ such that $\delta_{i_{0}}$ is a gem, we get $\delta(x, y)=0 \Rightarrow \forall j \delta_{j}(x, y)=0 \Rightarrow$ $\delta_{i_{0}}(x, y)=0 \Rightarrow x=y$.

(3) Each (generalized) pseudometric space induces a partition into the sets of points with zero distance and there is an induced (generalized) metric on the factor set. In fact, for a pseudogem space $(X, M, \delta)$ the relation $x \sim y \Leftrightarrow \delta(x, y)=0$ is an equivalence relation and the corresponding factorization yields a gem $\delta_{\sim}$ on $X / \sim$ defined simply by $\delta_{\sim}([x],[y])=$ $\delta(x, y)$. The situation is depicted by the commuting diagram

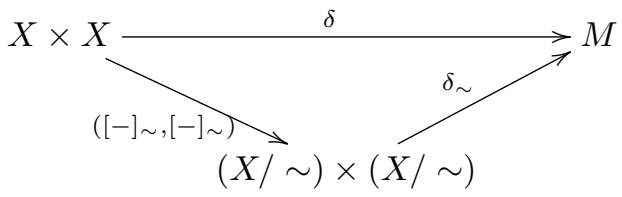

Here are some basic examples of gem and pseudogem spaces.

Example 2.3. (1) Gem spaces over $\underline{\mathbb{R}}$ are metric spaces.

(2) Gem spaces over $\underline{\mathbb{N}}$ are digital spaces. Indeed, each connected undirected graph yields a metric with values in $\underline{\mathbb{N}}$, which makes it a gem space over $\underline{\mathbb{N}}$. On the other hand, each gem $\delta$ over $\underline{\mathbb{N}}$ induces a reflexive symmetric relation $E=\{(u, v) \mid \delta(u, v) \leq 1\}$, thus we obtain a (undirected) graph with edges in $E$. Pseudogem spaces over $\underline{\mathbb{N}}$ will be called pseudodigital. 
(3) Due to the third property above, each pseudogem $\pi$ on $X$ is a composition $\delta \circ(\nu, \nu)$ of a mapping $\nu$ from $X$ into a gem space $Y$ and of the gem $\delta$ on $Y$, and vice versa. Thus every pseudogem space uniquely corresponds to a mapping into a gem space.

(4) For every pseudogem space $(X, M, \delta)$ there exists zero pseudogem $\tau_{X, M}$ : $X^{2} \rightarrow M$ given by $\tau(x, y)=0$.

Note 2.3.1. By $\mathrm{e}_{n}, \mathrm{~m}_{n}$ and $\mathrm{w}_{n}$ we denote the Euclidean, Manhattan and maximalistic metric, respectively, on a subset of $\mathbb{R}^{n}$.

For the purpose of a generalized setting, we introduce the following value algebras. As shown in $[15,18]$, given a commutative monoid $(M,+)$ satisfying

(1) $\forall a, b \quad a+b=0 \Rightarrow a=b=0$,

(2) $\forall a, b \exists ! c(a+c=b \vee b+c=a)$,

there is an induced linear order $\leq$ which makes $(M,+, \leq)$ an ordered commutative monoid. The order is defined by $a \leq b \Leftrightarrow \exists c(a+c=b)$. In the sequel, it will be called the induced order.

Definition 2.4. Let $(M,+)$ be a commutative monoid. If it satisfies the properties above, it is called self-symmetric. We call it archimedean if, for every $a, b \in M, a>0$, there exists $n \in \mathbb{N}$ such that $n a>b$. If the self-symmetric monoid has an infimum defined for each nonempty subset w.r.t. the induced order, we say that the monoid is self-consistent.

$(M,+)$ is called a meet-quantale if it is a complete lattice and the addition distributes over arbitrary meets.

We say that $(M,+)$ is metric, if it is a gem space over itself, i.e., there is a binary operation $\mu: M^{2} \rightarrow M$ (this notation will be used further on) satisfying strong self-uniformity, commutativity and $\triangle$-inequality.

Clearly, each self-consistent monoid has suprema defined for every bounded subset. A free meet-quantale over a value monoid $M$ is a set $\mathcal{W}(M)$ of expressions of the form $\bigwedge_{n \in N} n$ where $N \subseteq M$. It can be obtained as a set $\mathcal{U}(M)$ of upper sets where $\bigwedge_{n \in N} n=\uparrow N$ and the addition is given pointwise. The quantale multiplication of two upper sets $X, Y \subseteq M$ is defined as the upper closure of their algebraic product, i.e., $X \cdot Y=\uparrow\{x \cdot y \mid x \in X, y \in Y\}$ and meets are defined as intersections. There is a canonical embedding $\eta: M \rightarrow \mathcal{W}(M)$ given by $a \mapsto \uparrow a$; the mapping is antitone $(M, \leq) \rightarrow(\mathcal{U}(M), \subseteq)$, thus $\mathcal{W}(M)=(\mathcal{U}(M), \supseteq)$. See more on quantales in $[2,11,17,19,20]$ and $[12,13,21]$ for free algebras in general. The monoids in the definition are collectively called value monoids. One can find plenty of examples but we will use mostly only $(\underline{\mathbb{N}},+, 0, \leq,|-|)$ and $(\mathbb{R},+, 0, \leq,|-|)$ and denote them by the notation of the underlying set.

We recall from [15] some of the basic facts of value monoids:

Proposition 2.5. (1) Each self-symmetric monoid is a metric monoid with the inner gem called symmetric difference $\div$ given by

$$
a \div b=c \Leftrightarrow(a+c=b \vee b+c=a) \text {. }
$$


(2) Each atomic archimedean self-symmetric monoid is isomorphic to

$$
(\underline{\mathbb{N}},+, \leq) \text {. }
$$

(3) Each nontrivial self-consistent monoid is isomorphic to one of $\underline{\mathbb{N}}, \underline{\mathbb{R}}$.

As in each metric space, in every pseudogem space, we have a well defined concept of neighborhoods.

Definition 2.6. Given a point in a pseudogem space $\mathcal{X}=(X, M, \delta)$ and a scalar $a \in M$, the sets $O(x, a)=\{y \in X \mid \delta(x, y)<a\}$ and $B(x, a)=\{y \in X \mid$ $\delta(x, y) \leq a\}$ are called open and closed, respectively, $x$-centered ball of a radius a. $O(x, a)$ is also called an a-neighborhood of $x$. Moreover, we use the notation $O^{*}(x, a)=O(x, a) \backslash\{x\}$ for the corresponding punctured neighborhood and $B^{*}(x, a)=B(x, a) \backslash\{x\}$. The name of the dissimilarity can be denoted in the subscript of $O$ to distinguish neighborhoods for more pseudogems.

Definition 2.7. If $\mathcal{X}$ is a pseudogem space over metric monoid $(M, \mu)$, we say that a point $x$ in a gem space $(X, M, \delta)$ is isolated if there exists $e \in M$ such that $O_{\mu}^{*}(0, e) \neq \emptyset \wedge O_{\delta}^{*}(x, e)=\emptyset$. We say that a pseudogem space is dense if it contains no isolated points.

\section{2. $c$-cohesiveness}

The key concept of each theory of spaces carrying a geometric structure is that of continuity. In order to avoid misunderstanding due to some incompatibility issues, we use the concept of cohesiveness ([15]). It will play a role of graph homomorphisms for gem spaces over $\underline{\mathbb{N}}$ and, up to a certain extent, of continuous morphisms for (pseudo)gem spaces over $\underline{\mathbb{R}}$. Here we generalize the cohesiveness to a $c$-cohesiveness which enables a certain restricted discontinuity. From now on, let there be two pseudogem spaces $\mathcal{X}=(X, M, \pi)$ and $\mathcal{Y}=(Y, N, \rho)$ and consider $x \in X$ and $f: X \backslash\{x\} \rightarrow Y$.

Definition 2.8. Given $e \in N, L \in Y$ and $x \in X$, then we define the conditions of discontinuity restriction

$$
\begin{aligned}
& \left.D R_{1}(f, x, e, L) \Leftrightarrow\left(\exists d=d_{f, x, e, L} \in M\right)\left(\emptyset \neq f\left(O^{*}(x, d)\right) \subseteq O(L, e)\right)\right), \\
& Q(f, x, e, L) \Leftrightarrow\left(\emptyset \neq O^{*}(L, e) \rightarrow D R_{1}(f, x, e, L)\right) .
\end{aligned}
$$

Then we say

(1) $L$ is a $c$-limit of $f$ at the point $x$ iff $(\forall e>c) D R_{2}(f, x, e, L)$ and we write $\mathrm{\textrm {L }}(f, x, c, L)$,

(2) $L$ is a limit of $f$ at $x$ iff $(\forall e \in N) D R_{2}(f, x, e, L)$.

The set of all limits of $f$ in $x$ is denoted by $\lim _{x} f$. The map $f: \mathcal{X} \rightarrow \mathcal{Y}$ is said to be $(c-)$ cohesive at $x$ if $f(x)$ is a $(c$-)limit of $f$ at $x$. The map is said to be $(c-)$ cohesive if, it is $(c-)$ cohesive at every point of its domain.

Now we add some more requirements on the monoids and study related concepts. Firstly we show the following observation. 
Remark 2.9. (1) Given $L \in Y$ and $x \in X$, the set $\mathcal{H}=\{e \in N$ $\left.D R_{1}(f, x, e, L)\right\}$ is up-closed. Indeed, given $e>c, O(L, c) \subseteq O(L, e)$ and if $D R_{1}(f, c, x, L)$, then $\left.(\exists d \in M)\left(\emptyset \neq f\left(O^{*}(x, d)\right) \subseteq O(L, c)\right)\right)$ thus $f\left(O^{*}(x, d)\right) \subseteq O(L, e)$.

(2) If $N$ is self-consistent, then $e>\wedge \mathcal{H} \Leftrightarrow \exists(a \in \mathcal{H}) e>a$. Indeed, assume the converse, i.e., $(\forall a \in \mathcal{H}) e \leq a$, thus $e \leq \bigwedge \mathcal{H}$, which is a contradiction.

Definition 2.10. In a self-consistent monoid, we denote $S(0)=\bigwedge\{e \in N \mid$ $e>0\}$ the pseudo-successor of zero.

Clearly, if the monoid is $M$, then $S(0)= \begin{cases}0 & M=\underline{\mathbb{R}} \\ 1 & M=\underline{\mathbb{N}}\end{cases}$

Lemma 2.11. Let $L \in Y$ and $x \in X, N$ be self-consistent and $\rho$ be a gem. Then, the concept of a limit is equivalent to that of a $c$-limit for $c=S(0)$.

Proof. By definition, $L$ is a limit of $f$ at $x \Leftrightarrow(\forall e \in N) D R_{2}(f, x, e, L)$, which implies $(\forall e>S(0)) D R_{2}(f, x, e, L)$. On the other hand, $e>S(0)$ is by the property 2 above equivalent to $\exists a>0, e>a$, which is equivalent to $O_{\mu}^{*}(0, e) \neq \emptyset$. Assume $e \ngtr S(0)$, then $O_{\mu}^{*}(0, e)=\emptyset$. Assume $y \in O_{\rho}^{*}(L, e)$, then $0 \leq \rho(L, y)<e \Rightarrow$ $\rho(L, y) \in O_{\mu}(0, e) \Rightarrow \rho(L, y)=0$. If $\rho$ is a gem, then $\rho(L, y)=0 \Rightarrow L=y$, which is a contradiction, i.e. $O_{\rho}^{*}(L, e)=\emptyset$ and hence $D R_{2}(f, x, e, L)$ holds trivially. Thus $(\forall e \geq S(0)) D R_{2}(f, x, e, L) \Leftrightarrow(\forall e>0) D R_{2}(f, x, e, L)$, which proves the statement.

Remark 2.12. If $\rho$ is not strongly self-uniform, then $y$ in the proof above can be different from $L$ despite having zero distance. This can occur only if $N \cong \mathbb{N}$, i.e., $(Y, N, \rho)$ is a pseudodigital space such that $O_{\rho}^{*}(L, 0) \neq \emptyset$. If we have $(X, M, \pi)=$ $(Y, N, \rho)$ and $f\left(O^{*}(x, e)\right) \subseteq O^{*}(x, e)$ for every $e \geq 2$ and $f\left(O^{*}(x, 1)\right) \not \subseteq O^{*}(x, 1)$, then $f$ has a 1-limit $L$ at $x$ but not a limit.

The following lemma shows that, in some cases, the choice of $d_{t, e}$ in the discontinuity restriction can be done canonically.

Lemma 2.13. Let the assumptions of Definition 2.8 be satisfied and let $M$ be a self-consistent monoid. Given $e \in N, L \in Y$ and $x \in X$ such that $D R_{1}(f, x, e, L)$ is satisfied, then

$$
R_{x, e}=\left\{d>0 \mid \emptyset \neq f\left(O_{\pi}^{*}(x, d)\right) \subseteq O_{\rho}(L, e)\right\} .
$$

Then if $R_{t, e}$ is bounded, then it has a top element.

Proof. Since $D R_{1}(f, x, e, L)$ is satisfied, $D=R_{x, e} \neq \emptyset$. Assume $D$ is bounded and let $b=\sup D$. Since there exists $a \in D$, we have $a<b$ and $\emptyset \neq O_{\pi}^{*}(x, a) \subseteq$ $O_{\pi}^{*}(x, b)$, thus, $\emptyset \neq O_{\pi}^{*}(x, b)$. Let $y \in O_{\pi}^{*}(x, b)$, then $\pi(x, y)<b$. Thus, there exits $d \in D$ such that $\pi(x, y)<d$. Therefore, $y \in O_{\pi}^{*}(x, d)$, thus, $f(y) \in O_{\rho}(L, e)$. Thus, $f\left(O_{\pi}^{*}(x, b)\right) \subseteq O_{\rho}(L, e)$, hence $b \in D$.

This property can be useful when we work with self-consistent monoid - it enables us to define $d_{x, e}=\sup R_{x, e}$, the case of $D$ being unbounded has to be solved separately. 
Now we translate the $c$-cohesiveness and related concepts into the language of digital and metric spaces.

Theorem 2.14. A function $f$ between digital spaces with no isolated points is c-cohesive iff, for every $y$ adjacent to $x$, the distance between $f(x)$ and $f(y)$ is at most $c$.

Proof. Consider a digital space $(X, \underline{\mathbb{N}}, \pi)$.

" $\Rightarrow$ " Let $f$ be $c$-cohesive. Then, for every $x \in X, f(x)$ is a $c$-limit of $f$ at $x$, therefore, for every $e>c$ (i.e., $e \geq c+1),\left(\emptyset \neq O^{*}(f(x), e) \wedge(\exists d \in M)(\emptyset \neq\right.$ $\left.\left.f\left(O^{*}(x, d)\right) \subseteq O(f(x), e)\right)\right)$. Let $e=c+1$ and assume $O^{*}(f(x), c+1) \neq \emptyset$, i.e., namely $c \geq 1$. Then by the discontinuity restriction property $D R_{2}(f, x, e, L)$ we have $\emptyset \neq O^{*}(x, d)$ (which means that $d \geq 2$ ) and $f\left(O^{*}(x, d)\right) \subseteq O(f(x), c+1)$ ), which yields that each element $y$ adjacent to $x$ (thus, at the distance from $x$ smaller than $d$ ) is mapped to the distance smaller than $c+1$ from $f(x)$, i.e., $\rho(f(x), f(y)) \leq c$.

" $\Leftarrow$ " Let the second condition be satisfied. Then for every $x \in X$ and $e>c$ such that $\emptyset \neq O^{*}(f(x), e)$ there exists $z \in O^{*}(f(x), e)$, i.e. (by strong self-uniformity), $0<\rho(f(x), z)<e$, hence $e>1$. Let $d=2$. Then $O^{*}(x, 2)$ is a set of all elements adjacent to $x$, which is nonempty by the assumption of the lemma. Moreover for every $y \in O^{*}(x, 2)$, by the assumption of this part of the proof, $\rho(f(x), f(y)) \leq$ $c<e$, thus, $f(y) \in O(f(x), e)$. This means that $f(x)$ is a $c$-limit of $f$ at $x$.

Theorem 2.15. A function $f$ from a metric space to $(\mathbb{R}, \mathbb{R}, \mu)$ (here $\mu$ is the absolute value of the difference) has a c-limit $L$ at $x_{0}$ iff $L_{S}, L_{I} \in B_{\mu}(L, c)$ where $L_{S}=\lim \sup _{x \rightarrow x_{0}} f(x), L_{I}=\lim \inf _{x \rightarrow x_{0}} f(x)$.

Proof. Let $f: X \rightarrow \underline{\mathbb{R}}$ satisfy the assumptions of the statement, let $L_{S}(d)=$ $\bigvee f\left(O^{*}\left(x_{0}, d\right)\right), L_{I}(d)=\bigwedge f\left(O^{*}\left(x_{0}, d\right)\right)$ and $L_{S}=\lim _{d \rightarrow 0} L_{S}(d)$ and $L_{I}=\lim _{d \rightarrow 0} L_{I}(d)$.

" $\Rightarrow$ " Let $f$ have a $c$-limit $L$ at $x$. Since $\underline{\mathbb{R}}$ does not contain isolated points, we have in general $O^{*}(y, e) \neq \emptyset \Leftrightarrow e>0$. Given $e>c$, by the property of $c$-limit there exists $d_{e}>0$ ( $\operatorname{such}$ that $d_{e} \rightarrow 0$ for $e \rightarrow 0$ ) satisfying $f(x) \in O^{*}(L, e)$ for $x \in O^{*}\left(x_{0}, d_{e}\right)$, thus, $f(x)<L+e$. Moreover, the function $L_{S}(d)$ is increasing for $d>0$. Therefore,

$$
\begin{aligned}
& L_{S}=\lim _{d \rightarrow 0} \bigvee f\left(O^{*}\left(x_{0}, d\right)\right) \leq \lim _{e \rightarrow c} \bigvee f\left(O^{*}\left(x_{0}, d_{e}\right)\right) \\
& \leq \lim _{e \rightarrow c} \bigvee(L+e) \leq \\
& L+c .
\end{aligned}
$$

Therefore, $\mu\left(L, L_{S}\right) \leq c$ and $L_{S} \in B_{\mu}(L, c)$. The proof for $L_{I} \in B_{\mu}(L, c)$ is analogous.

" $\Leftarrow$ " Let the second condition be satisfied. If $L_{S}=L_{I}$, then the limit exists and $L=L_{S}$, thus, the condition $L_{S}, L_{I} \in B(L, c)$ gives no restriction on $c$. However, by Lemma 2.11, $L$ is a $c$-limit for $c=S(0)$. Moreover, for $c=0$ we obtain $O^{*}(L, c)=\emptyset$ thus, $D R_{2}\left(f, x_{0}, 0, L\right)$ is satisfied.

Now assume $L_{S} \neq L_{I}$, i.e., $L_{S}>L_{I}$. Let $\lambda=\frac{L_{S}-L_{I}}{2}$. Since $L_{S}, L_{I} \in B(L, c)$, $\mu\left(L_{S}, L\right) \leq c, \mu\left(L_{I}, L\right) \leq c$, thus, $\mu\left(L_{S}, L_{I}\right) \leq 2 c$ and since $\mu\left(L_{S}, L_{I}\right)=L_{S}-L_{I}$ we get $c \geq \lambda$ and $L \in B\left(L_{S}, c\right) \cap B\left(L_{I}, c\right)$. 
Let $e>c, a=e-c$. We will show the property $D R_{1}\left(f, x_{0}, e, L\right)$. Since $L_{S}$ and $L_{I}$ are obtained as limits there exists $d_{a}>0$ such that, for every $u \in O\left(0, d_{a}\right), \mid L_{S}-$ $L_{S}(u) \mid<a$ and $\left|L_{I}-L_{I}(u)\right|<a$. Let $0<b<d_{a}$. Then, for every $x \in B^{*}\left(x_{0}, b\right) \subseteq$ $O^{*}\left(x_{0}, d_{a}\right), L_{I}(b) \leq f(x) \leq L_{S}(b)$, i.e., $f\left(B^{*}\left(x_{0}, b\right)\right) \subseteq\left[L_{I}(b), L_{S}(b)\right]$. We will show that both boundaries of the interval belong to $O(L, e)$. To show $L_{I}(b) \in O(L, e)$ we need $\left|L_{I}(b)-L\right|<e$. Recall that $\left|L-L_{I}\right| \leq c,\left|L_{I}-L_{I}(b)\right|<a$ and $a+c<e$. Therefore, $\left|L_{I}(b)-L\right|=\left|L_{I}(b)-L_{I}+L_{I}-L\right| \leq\left|L_{I}(b)-L_{I}\right|+\left|L_{I}-L\right|<a+c<e$. Thus, $L_{I}(b) \in O(L, e)$. The proof of $L_{S}(b) \in O(L, e)$ is analogous. Thus, we have $\left[L_{I}(b), L_{S}(b)\right] \subseteq O(L, e)$, hence $f\left(O^{*}\left(x_{0}, b\right)\right) \subseteq f\left(B^{*}\left(x_{0}, b\right)\right) \subseteq O(L, e)$ and $L$ is a $c$-limit of $f$ at $x$.

Other instances of $c$-cohesiveness can be seen in the following examples. In each case, we mention the least value of cohesiveness (if it exists).

Example 2.16. (1) The cohesiveness of a mapping between metric spaces coincides with the usual concept of continuity, if the spaces contain no isolated points.

(2) The $c$-cohesiveness enables a vertical jump by $c$ both up and down (in the graph of the function). Thus, e.g., the floor function $f l:\left(\mathbb{R}, \mathbb{R}, \mathrm{e}_{1}\right) \rightarrow$ $\left(\mathbb{R}, \underline{\mathbb{R}}, \mathrm{e}_{1}\right)$ is 1 -cohesive. The function

$$
x \mapsto\left\{\begin{array}{rll}
\lfloor x\rfloor & \text { for } & x \notin \mathbb{Z} \\
x-\frac{1}{2} & \text { for } & x \in \mathbb{Z}
\end{array}\right.
$$

is $\frac{1}{2}$-cohesive.

(3) The floor function can be considered also as $f:\left(\mathbb{R}, \underline{\mathbb{R}}, \mathrm{e}_{1}\right) \rightarrow\left(\mathbb{Z}, \underline{\mathbb{N}}, \mathrm{m}_{1}\right)$. Let $x \in \mathbb{R}$ and $e \in \mathbb{N}$. If $e<2$, then $O^{*}(f(x), e)=\emptyset$. If $e \geq 2$, then $f(x)+1 \in O^{*}(f(x), 2)$. Then for $d=1 \in \mathbb{R}$ we have $\emptyset \neq(x-1, x+1) \backslash\{x\}$ and for every $y \in \neq(x-1, x+1) f(y) \in[f(x)-1, f(x)+1]=O(f(x), 2)$. Therefore, $f$ is cohesive.

(4) Let $(X, M, \pi)=(Y, N, \rho)=\left(\mathbb{R}, \underline{\mathbb{R}}, \mathrm{e}_{1}\right)$ and let $f(x)=\sin \frac{1}{x}$. While this function has no value and no limit at $x=0,0$ is its 1 -limit at 0 . One can see that $\lim \sup f(x)=1$ and $\liminf f(x)=-1$ and $1,-1 \in[L-c, L+c]$ iff $c \geq 1$, in which case $L=0$. Thus, $\mathrm{E}(f, 0,1,0)$, by Theorem 2.15 .

(5) The mapping $f: \mathbb{R}^{2} \rightarrow \mathbb{Z}^{2}$ given by collapse of each half-open square $A=[a, a+1) \times[b, b+1)$ with $a, b \in \mathbb{Z}$ to a point $[a, b] \in \mathbb{Z}^{2}$ is cohesive $\left(\mathbb{R}^{2}, \underline{\mathbb{R}}, \mathrm{e}_{n}\right) \rightarrow\left(\mathbb{Z}^{2}, \underline{\mathbb{N}}, \mathrm{w}_{n}\right)$. Indeed, for $e \in \underline{\mathbb{N}}$ with $O_{\mathrm{w}}^{*}([a, b], e) \neq \emptyset$, i.e., $e \geq 2$, we have, for $d=\frac{1}{2}, O_{\mathrm{e}}^{*}([a, b], e) \neq \emptyset$, and $\forall[x, y] \in A$ and $\forall[u, v] \in$ $O_{\mathrm{e}}^{*}([x, y], d)$ we have $f(u, v)=[p, q] \in[a-1, a+1] \times[b-1, b+1]$, thus, $\mathrm{w}([p, q],[a, b]) \leq 1<2$.

(6) Cohesive mapping from a space over a nontrivial monoid to a space with a zero pseudogem is an arbitrary mapping. Indeed, each open ball w.r.t. the zero pseudogem is the whole space itself, thus, the cohesiveness is trivially satisfied. 


\subsection{Defect of cohesiveness}

From now on, let there be two pseudogem spaces $\mathcal{X}=(X, M, \pi), \mathcal{Y}=(Y, N, \rho)$ over self-consistent monoids and consider $f: X \backslash\{x\} \rightarrow Y$.

Definition 2.17. Given $x \in X$ and $L \in Y$, then we define defect of the limit $L$ of $f$ at $x$ as

a scalar

$$
D(f, x, L)=\inf \left\{e \in N \mid D R_{1}(f, x, e, L)\right\} .
$$

If $f$ is defined in $x$, then we put $D(f, x)=D(f, x, f(x))$ and $D(f)=\sup \{D(f, x) \mid$ $x \in X\}$ be the defect of the function $f$.

The defect of a function evaluates the failure of function's cohesiveness. It may not be defined if the set $S=\{D(f, x) \mid x \in X\}$ is not bounded. However, if $f$ is bounded, i.e., there exists $b \in N$ such that $\sup \{f(u) \div f(v) \mid u, v \in X\} \leq b$, then $D(f, x) \leq \sup \{f(u) \div f(v) \mid u, v \in X\} \leq b$.

Lemma 2.18. Under the assumptions above, $E(f, x, D(f, x, L), L)$ and if $\mathcal{Y}$ is dense, then $D(f, x, L)=\min \{e \in N \mid E(f, x, e, L)\}$

Proof. In the proof we simplify the notation by dropping $f, x$ and $L$ from the parameter lists of $\mathrm{E}, H, Q$. We will show $\mathrm{L}(b)$ for $b=D(f, x, L)$. Let $\mathcal{H}=\{c \in$ $\left.N \mid D R_{1}(c)\right\}$. We proceed backwards:

$$
\begin{aligned}
\mathrm{E}(b) & \Leftrightarrow \quad(\forall e>b) D R_{2}(e) \\
& \Leftrightarrow \quad(\forall e>\inf \mathcal{H})\left(O^{*}(L, e) \neq \emptyset \Rightarrow D R_{1}(e)\right. \\
& \Leftrightarrow \quad(\forall e)\left((\exists a \in \mathcal{H}) a<e \Rightarrow\left(O^{*}(L, e) \neq \emptyset \Rightarrow D R_{1}(e)\right)\right)
\end{aligned}
$$

But $(\exists a \in \mathcal{H}) a<e \Rightarrow\left(O^{*}(L, e) \Rightarrow D R_{1}(e)\right)$ is clearly satisfied since $\mathcal{H}$ is an upper set by Remark 1. Thus, $\mathrm{E}(b)$ is satisfied. Thus, $b \in\{e \in N \mid \mathrm{E}(e)\}$. Now assume $\mathrm{E}(c)$ for $c>0$ and $\mathcal{Y}$ be dense. Then $(\forall e>c)\left(O^{*}(L, e) \neq \emptyset \Rightarrow D R_{1}(e)\right)$. If $D R_{1}(e)$, then $e \geq b$. Since $0<c<e$ and $L$ is not isolated, $O^{*}(L, e) \neq \emptyset$ thus, $D R_{1}(e)$, therefore $e \geq b$ by the definition of the defect. Thus, $b$ is a lower bound for $\{e \in N \mid \mathrm{E}(e)\}$ and since we have proved that it belongs to it, it is its least element.

\subsection{Paths in gem spaces}

One concept common to the theory of metric and topological spaces and graph theory is that of paths and path-connectedness. We work with a self-symmetric monoid $M$ with the induced metric $\mu$ given by symmetric difference. Now we can define paths in a pseudogem space.

Definition 2.19. Let $(X, M, \delta)$ be a pseudogem space over a self-symmetric monoid. Given a pair of points $x, y \in X$, then a path $\gamma: x \rightarrow y$ is a mapping $\gamma: M \rightarrow X$ with the properties:

- $\gamma:(M, \mu) \rightarrow(X, \omega)$ is cohesive,

- $\gamma(0)=x$,

- $(\exists m=m(\gamma) \in M) \gamma^{-1}(y)=\uparrow m(\gamma)$. 
The value $\gamma(m(\gamma))$ will be denoted $\gamma(\infty)$. We say that the space is path-connected if any two points can be connected by a path. The set of all paths $x \rightarrow y$ is denoted by $\Gamma(x, y)$ and we may use the notation of pseudogem under the arrowhead such as $x \rightarrow \delta$ for emphasis.

Replacing the condition of cohesiveness of $\gamma$ by the $c$-cohesiveness we obtain a weaker concept of a c-path.

Remark 2.20. As shown in [15], the self-symmetry enables the following usual operations of paths:

- trivial paths (constant maps) on each point,

- inverse path (path with the same image with swapped endpoints),

- concatenation of paths (endpoint of the first is the beginning point of the second; the image of the concatenated path is the union of the images of the original paths),

- subpaths (each path is a concatenation of subpaths).

Even if we have avoided precise definitions, it will be sufficient to know these properties in order to understand their applications. These concepts will be important namely in the context of valuations of a topographic path.

Example 2.21. (1) Each curve in a metric space with endpoints $x$ and $y$ is a path $x \rightarrow y$, thus, the path-connectedness has its usual (topological) meaning.

(2) In a digital space, each path is a walk in the graph-theoretical meaning, the mapping maps 0 to the initial vertex and preserves successorship and remains constant up from certain value.

(3) In both cases, $c$-paths differ from the paths by permitting disconnection by distance $c$.

(4) Path w.r.t. the zero pseudogem $\tau_{(X, M)}$ is any mapping $\gamma: M \rightarrow X$ such that there exists $m \in M$ with $\gamma(\uparrow m)=\{\gamma(m)\}$, i.e., $\tau_{X, \mathbb{N}}$ is any finite sequence of points in $X$.

The digital space $\mathcal{Z}_{k}=\left(\mathbb{Z}^{k}, \underline{\mathbb{N}}, \delta\right)$ with some adjacency-induced metric is path connected iff it is connected w.r.t. the adjacency relation. We use namely 4adjacency $\alpha_{+}$and 8-adjacency $\alpha_{\square}$ (and their more-dimensional analogies with the dimension denoted by upper script). Namely $\left(\mathbb{Z}^{k}, \underline{\mathbb{N}}, \mathrm{m}_{k}\right)$ and $\left(\mathbb{Z}^{k}, \underline{\mathbb{N}}, \mathrm{w}_{k}\right)$ is defined by $\alpha_{+}^{k}$ and $\alpha_{\square}^{k}$, respectively.

Path length. To formulate the length of a path in a gem space, we will need the archimedean property. From now on, let $\mathcal{X}=(X, M, \delta)$ be a pseudogem space over an archimedean self-symmetric monoid. Let $\gamma: x \rightarrow-y$ be a path in $\mathcal{X}$. Let $a=m(\gamma)$, then, given $c \in M$, the set $K_{c, a}=\{k \in \underline{\mathbb{N}} \mid k c \geq a\}$ is nonempty by the archimedean property (Lemma 2 ), thus, there exists $a / c=\min K_{c, a}$. Let

$$
q(c)=\sum_{k=0}^{a / c} \delta(\gamma(k c), \gamma((k+1) c),
$$


we obtain a mapping $q: M \rightarrow M$.

Definition 2.22. Consider the space $\mathcal{X}$ above. If $M$ is a non-atomic monoid, then we define for a path $\gamma: x \rightarrow-\rightarrow$ its length as

$$
l(\gamma)=\lim _{c \rightarrow 0} q(c)
$$

if the limit exists.

The following properties are shown in [15]:

Proposition 2.23. (1) If the limit from Definition 2.22 exists, then it is unique.

(2) The length of a path corresponds to the curve length in metric space.

(3) Length of a walk (i.e., of its sequence of edges) in a digital space can be expressed as the above limit.

Thus, the limit provides a tool to measure the path lengths for any archimedean self-symmetric monoid; in the case of an atomic monoid we choose the limit which corresponds to the length of the corresponding edge sequence. Since the limit is strongly dependent on the gem used for the calculation, we will often denote it in the subscript of $l$. A path whose length is defined and finite is called rectifiable ([4]). A path-connected gem space is called rectifiable if for each two points there exists a connecting rectifiable path.

Lemma 2.24. Let $\gamma: x \rightarrow y$ be a path in $\mathcal{Z}_{k}$ with the metric $\delta=\mathrm{m}_{k}$. Then $l_{\delta}(\gamma) \equiv_{2} \delta(x, y)$, i.e., $(\exists k \in \underline{\mathbb{N}}) l_{\delta}(\gamma) \div \delta(x, y)=2 k$.

Proof. We proceed by induction over length of the path. If $l_{\delta}(\gamma)=0$, then $\gamma$ is trivial $x \rightarrow x$, and $\delta(x, x)=0$ and $0 \equiv_{2} 0$. Now let $\gamma: x \rightarrow y$ and assume $l_{\delta}(\gamma) \equiv_{2} \delta(x, y)$. Since each path is a walk, it can be created as a sequence of adjacent pairs o points. Consider $z$ adjacent to $y$ and consider extension $\gamma^{\prime}$ of $\gamma$ by $z$. Clearly $\gamma^{\prime}$ is a path in $\mathcal{Z}_{k}$ and $\mathrm{m}_{k}(x, z)=\mathrm{m}_{k}(x, y) \pm 1$. Indeed, due to Manhattan metric and discreteness of $\underline{\mathbb{N}}$, there is no point whose distances to $y$ and $z$ are the same, thus, $\mathrm{m}_{k}(x, y)$ is either greater by 1 or smaller by one. In each case, we have $l_{\delta}\left(\gamma^{\prime}\right)=l_{\delta}(\gamma)+1 \equiv \delta(x, y)+1 \equiv_{2} \mathrm{~m}_{k}(x, z)=\delta(x, z)$.

Spaces with multiple gems. Let $\mathcal{X}_{1}=\left(X, M_{1}, \delta_{1}\right)$ and $\mathcal{X}_{2}=\left(X, M_{2}, \delta_{2}\right)$ be two pseudogem spaces on the same set of points. Let $M_{1}$ be an archimedean selfsymmetric monoid. Then one may consider paths on $X$ with respect to $X_{1}$ with the interest of length with respect to the pseudogem $\delta_{2}$. This can be expressed, for a path $\gamma$ in $\mathcal{X}_{1}$, as

$$
\delta_{1} l_{\delta_{2}}(\gamma)=\lim _{c \rightarrow 0, c \in \mathcal{M}_{1}} \sum_{k=0}^{m(\gamma) / c} \delta_{2}(\gamma(k c), \gamma((k+1) c)
$$

where $m(\gamma) / c=\max \{k \in \underline{\mathbb{N}} \mid k c \geq m(\gamma)\}$ exists due to the archimedean property (Lemma 2) of $M_{1}$. The left subscript of $l$ can be omitted. 
Example 2.25. (1) Let $X=\mathbb{Z}^{3}, M_{1}=\underline{\mathbb{N}}, M_{2}=\underline{\mathbb{R}}, \delta_{1}=\mathrm{w}_{3}$ (maximalistic metric) and $\delta_{2}=\mathrm{e}_{3}$ (Euclidean metric). Consider a w3-path $\gamma:[0,0,0]-\rightarrow$ $[1,1,0]$ given by the walk $[0,0,0]-[1,0,0]-[0,1,1]-[1,1,0]$. Then $l(\gamma)=$ $l_{\mathrm{w}_{3}}(\gamma)=3$ and $l_{\mathrm{e}_{3}}(\gamma)=1+\sqrt{3}+\sqrt{2}$.

(2) Consider $(X, \underline{\mathbb{N}}, \delta)$ and its zero pseudogem $\tau=\tau_{X, \mathbb{N}}$. Then for a $\tau$-path $\gamma$ we have $l_{\delta}(\gamma)=\sum_{i=1}^{m(\gamma)} \delta(\gamma(i-1), \gamma(i))$ while $l_{\tau}(\gamma)=0$.

Inner gem and its abstraction. Now we show a very general construction of pseudogems. It involves a (weak) path-valuation. It is any mapping $J: \bigcup\{\Gamma(x, y) \mid$ $x, y \in X\} \rightarrow N$ to an ordered monoid $N$ which satisfies:

- it assigns the bottom element to a path iff the path is trivial (only " $\Leftarrow$ " for the weak version),

- the assignment is independent of the path direction (i.e., the path has the same image as its inverse),

- the assignment is sub-additive w.r.t. the concatenation of the paths.

If we want to emphasize that path-valuation is not only a weak path valuation, we say that it is a strict path-valuation.

Example 2.26. (1) The paradigmatic path valuation is the path length $l$. It is easy to see that the properties are satisfied even for ${ }_{\pi} l_{\rho}$ if we have two gems. If $\rho$ is only a pseudogem, we obtain a weak path valuation.

(2) For every (pseudo)gem $\delta$, the mapping $s_{\delta}(\gamma)=\delta(\gamma(0), \gamma(\infty))$ is a (weak) path-valuation.

Now we show two important properties. The proof of the first is in [15] and of the second is analogous.

Definition 2.27. Let $J$ be a (weak) path valuation. Then by assignment

$$
\omega_{J}(x, y)=\bigwedge_{\gamma: x-\rightarrow y} J(\gamma)
$$

we define abstract $J$-induced pseudogem.

Proposition 2.28. Let $(X, M, \rho)$ be a (pseudo)gem space over an ordered monoid and $J$ be a path-valuation with values in a monoid $N$, then the mapping $\omega_{J}$ defines a (pseudo)gem space $\left(X, \mathcal{W}(N), \omega_{J}\right)$. If $J$ is only a weak path-valuation, then $\omega_{J}$ is a pseudogem.

Before we show some examples, we compare this abstract expression with a concrete one, defined only in a self-consistent monoid $N$.

Definition 2.29. Let $J$ be a (weak) path valuation. Then by the assignment

$$
\lambda_{J}(x, y)=\inf \{J(\gamma) \mid \gamma: x \rightarrow y\}
$$

we define (concrete) $J$-induced pseudogem.

In general, we have this property:

Theorem 2.30. Let $\delta$ be a pseudogem on $X$, then the $J$-induced gem $\lambda_{J}$ is also a pseudogem. 
Out of the main line of research, we mention the following relationship of these two induced pseudogems. Recall from [10] that given two maps $\alpha: A \rightarrow B$ and $\beta: C \rightarrow B$ to a poset, then a mapping $\gamma: A \rightarrow C$ is right Kan extension of $\alpha$ along $\beta$ (we write $\gamma=\operatorname{Ran}_{\beta} \alpha$ ) if $\beta \gamma \leq \alpha$ and for every $\delta: A \rightarrow C, \beta \delta \leq \alpha$ implies $\delta \leq \gamma$.

Theorem 2.31. Let $\mathcal{X}=(X, M, \delta)$ be a pseudogem space over a self-consistent monoid and $J$ be a weak path-valuation. Then

$$
\lambda_{J}=\operatorname{Ran}_{\eta} \omega_{J}
$$

where $\eta: M \rightarrow \mathcal{W}(M)$ is the canonical embedding.

Proof. It is easy to see that $\eta \lambda_{J} \leq \omega_{J}$. Indeed, given a pair of points $x, y \in X$, then $\lambda_{J}(x, y)=\inf \{J(\gamma) \mid \gamma: x \rightarrow y\} \leq J(\gamma)$ for every $\gamma: x \rightarrow y$. Thus,

$$
\begin{aligned}
\eta \lambda_{J}(x, y) & =\uparrow \lambda_{J}(x, y) \\
& \leq \uparrow J(\gamma)
\end{aligned}
$$

thus, $\eta \lambda_{J}(x, y) \leq \bigwedge_{\gamma} \uparrow J(\gamma)=\bigwedge_{\gamma: x \rightarrow-\rightarrow} J(\gamma)=\omega_{J}(x, y)$. Now assume that there exists $\rho: X^{2} \rightarrow M$ such that $\eta \rho \leq \omega_{J}$. Then

$$
\begin{aligned}
\eta \rho(x, y) & \leq \omega_{J}(x, y) & \Rightarrow \\
\uparrow \rho(x, y) & \leq \bigwedge_{\gamma} \uparrow J(\gamma) & \Rightarrow \\
(\forall \gamma: x \rightarrow y) & \rho(x, y) \leq J(\gamma) & \Rightarrow \\
\rho(x, y) & \leq \inf \{J(\gamma) \mid \gamma: x-\rightarrow y\} & \Rightarrow \\
\rho(x, y) & \leq \lambda_{J}(x, y) &
\end{aligned}
$$

thus, $\rho \leq \lambda_{J}$.

Remark 2.32. In some cases we need to modify the defining set of paths for $\lambda$ or $\omega$. E.g., for a path length we need $\gamma$ to be rectifiable paths. However, if $\mathcal{X}$ is a rectifiable space, then the set is always nonempty and induces

$$
\lambda_{l}(x, y)=\inf \{l(\gamma) \mid \gamma: x \rightarrow y\}
$$

One can show that it is indeed a gem. In the case of a digital space, the inner gem is fully determined by the spanning tree on $X$ with edges representing the pairs of the distance equal to 1 .

In the case of $J=l$ we drop the notation of path valuation and we obtain abstract and concrete inner gem $\omega$ and $\lambda$, respectively. It is clear that, in general, $\delta \leq \lambda_{l_{\delta}}$. Moreover, as shown in the following example, the gem $\omega$ is slightly more perceptive than $\lambda$.

Example 2.33. Consider a set $\mathbb{R}^{2} \backslash(0,1) \times\{0\}$ as a subspace of the gem space $\left(\mathbb{R}^{2}, \mathbb{R}, \mathrm{e}_{2}\right)$. Consider points $x=[0,0]$ and $y=[1,0]$. Then clearly $\lambda(x, y)=$ $\inf _{\gamma: x \rightarrow y} l(\gamma)=1=\mathrm{e}_{2}(x, y)$ (we can make a sequence of paths $x \rightarrow y$ whose length converge to 1$)$. However $\omega(x, y)=\bigwedge_{\gamma: x \rightarrow \rightarrow y} l(\gamma)>\bigwedge\{t \mid t \geq 1\}=\eta(1)$. Therefore, the canonical embedding $\eta$ maps $\eta \lambda(x, y)<\omega(x, y)$. Thus, $\omega$ distinguishes between the distances $([0,0],[1,0])$ and $([0,0],[0,1])$ despite their values of $\lambda\left(\right.$ and $\left.\mathrm{e}_{2}\right)$ in $\underline{\mathbb{R}}$ are the same. 
Example 2.34. Given a space with two pseudogems $\pi, \rho$, the assignment on the $\pi$-paths $\gamma \mapsto l_{\rho}(\gamma)$ yields a path valuation. The corresponding abstract gem is $\omega_{l_{\rho}}(x, y)=\bigwedge_{\gamma: x \rightarrow \rightarrow_{\pi} y} l_{\rho}(\gamma)$.

(1) In the case of $\pi=\rho$, we obtain the abstract inner gem.

(2) For $M=\underline{\mathbb{N}}, \pi=\tau_{X, \underline{\mathbb{N}}}$ and $J={ }_{\tau} l_{\rho}$ we get generalized connectedness term $\kappa(x, y)=\omega_{J}(x, y)=\bigwedge_{\gamma \in \Gamma_{\tau}(x, y)} \sum_{i=1}^{m(\gamma)} \rho(\gamma(i-1), \gamma(i))$, used e.g., in the image processing for determining the value of closeness of two pixels in the digital image, see [13]. The original approach of fuzzy affinity [7] employs similarity of connectedness function, which is a dual to $\lambda_{\alpha}$ for a particular similarity $\alpha$ (a pixel affinity).

(3) Due to Proposition 2, we have pseudogems $(\pi, \rho),\left(\pi, \omega_{J}\right),\left(\pi, \rho, \omega_{J}\right)$, etc. Thus, if we consider, e.g., $h=(\pi, \rho): X^{2} \rightarrow M \times M$, then $\omega_{h}: X^{2} \rightarrow$ $\mathcal{W}\left(M^{2}\right)$ assigns, to a pair $x, y$, a formal meet of sum of pair-values $(\pi(u, v), \rho(u, v))$ along all paths which are undominated in the sense of bicriterial comparison. This will be studied in context of topographic characteristics of paths.

Remark 2.35. Obviously, a repetition of the inner gem production is idempotent, i.e., $l_{\lambda_{l}}=l$. Indeed, since $\delta \leq \lambda_{l_{\delta}}$, we get $l_{\lambda_{l}} \geq l$. Now consider a path $\gamma: x \rightarrow y$ in $\mathcal{X}$. Then

$$
\begin{aligned}
l_{\lambda_{l}}(\gamma) & =\lim _{c \rightarrow 0} \sum_{k=0}^{m(\gamma) / c} \lambda_{l}(\gamma(k c), \gamma((k+1) c)) \\
& \left.=\lim _{c \rightarrow 0} \sum_{k=0}^{m(\gamma) / c} \inf \{l(\beta) \mid \beta: \gamma(k c) \rightarrow \gamma((k+1) c))\right\} \\
& \left.\leq \lim _{c \rightarrow 0} \sum_{k=0}^{m(\gamma) / c} \inf \left\{l\left(\gamma_{k c,(k+1) c}\right): \gamma(k c) \rightarrow \gamma((k+1) c)\right)\right\} \\
& =\lim _{c \rightarrow 0} l(\gamma) \\
& =l(\gamma)
\end{aligned}
$$

hence $l_{\lambda_{l}}=l$.

Single point characteristics. In some cases we will need a characteristic of a point which reflects the properties of some subspace. It can be expressed as a distance of the point from a set. Such a characteristic can be either concrete or abstract as in the previous section.

Definition 2.36. Consider a pseudogem space $(X, M, \delta)$ over a self-consistent monoid and a subset $Y \subseteq X$. Let $x \in X$. We define the distance from $x$ to $Y$ as

$$
\widetilde{\delta}(x, Y)=\inf \{\delta(x, y) \mid y \in Y\}
$$

and its abstract version

$$
\widehat{\delta}(x, Y)=\bigwedge\{\delta(x, y) \mid y \in Y\}
$$


Again, we can see that $\eta \widetilde{\delta}(x, Y) \leq \widehat{\delta}(x, Y)$ and $\widehat{\delta}(-, Y)$ is a more perceptive quantity than $\widetilde{\delta}(-, Y)$. Moreover, note that if $Y$ is empty, then $\widetilde{\delta}(x, Y)$ may not be defined. The non-emptiness property has to be always verified when working with these characteristics.

\section{TOPOGRAPHIC SPACES}

In the theory of digital terrain modeling, topographic spaces are characterized by several attributes such as strict local maxima and minima, saddle points, ridge points, plane points etc. [16] All of them are determined by the function of elevation. The digital data for DTM usually have their origin in the real space and are obtained transformed by the process of acquisition. If we define properly the topographic space with a sufficient level of generality, we may use the unified approach for both original and resulting situation. Then it makes sense to compare methods of data acquisition and processing by preservation of the attributes in question.

In order to define a topographic space, we need an elevation function. We will work with a pseudogem space $(X, M, \delta)$ over a self-symmetric monoid. Then we may use $M$ as a value set for the elevation function. However, in contrast to what one would expect in the Euclidean space, we do not generally assume the elevation to be continuous or cohesive, these requirements would be too strict.

Thus, we can define:

Definition 3.1. A topographic space is a pair $(\mathcal{X}, h)$ consisting of a pseudogem space $\mathcal{X}=(X, M, \delta)$ over a self-symmetric monoid and an elevation function, which is a mapping $h: X \rightarrow M$.

Example 3.2. Examples of topographic spaces can be found almost everywhere, since a general situation in which "objects are positioned and have some value" yields the example of a topographical space: the only restriction is that the values are nonnegative. Thus, we can take in account e.g. any physical positively real valued quantity distributed over a metric space (such as a height of the terrain over a sealevel or a pressure of a liquid in a closed system) or over its (possibly finite) subset (height of trees, population of a city with a given location, price of fuel on gas stations, etc.) Besides that, very important example is a digital gray scale image. It is a set $X \subset \mathbb{Z}^{2}$ and the elevation is the grayness function mapping $X$ to a subset of $\underline{\mathbb{N}}$.

While the elevation function may not have to be cohesive, we can ask for some level of its cohesiveness along a chosen path. From now on, let $\mathcal{X}=(X, M, \delta)$ be a pseudogem space over a self-symmetric monoid and $(\mathcal{X}, h)$ be a topographic space.

Definition 3.3. For $c \in M$, we say that a path $\gamma$ in $\mathcal{X}$ is passable / c-passable / horizontal, if the composition $h \circ \gamma$ is cohesive / c-cohesive / constant, respectively. The same adjectives refer to the space $(\mathcal{X}, h)$ if all of its points can be pairwise connected by a path of the given kind, and the points are then called mutually accessible, c-accessible or isohyptical, respectively. 
We define weaker versions of the above properties: points $x, y \in X$ are mutually nearly accessible if they are $c$-accessible for every $c>0$. A space is called nearly passable if all its points are pairwise mutually nearly accessible.

Due to Lemma 2.11, each passable space is $S(0)$-passable, which implies $c$ passablity for every $c \geq S(0)$, thus, for every $c>0$. Hence, $c$-passablity is generally weaker condition for passability but equivalent in the case of digital spaces. The study of these properties of spaces with non-cohesive elevation are our further goal.

\subsection{Elevation defect along a path}

Now we consider a topographic space $(\mathcal{X}, h)$ over a self-consistent monoid. The rectifiable paths in $\mathcal{X}$ enable the definition of another path valuation.

Lemma 3.4. Let $h$ be bounded on every bounded subset of $X$. Then $D(h \gamma)$ is defined for every rectifiable path $\gamma$.

Proof. Since $\gamma$ is rectifiable, for every $t \in M, \delta(\gamma(0), \gamma(t)) \leq l\left(\gamma_{0, t}\right) \leq l(\gamma)$ is finite, thus, there is a bounded set $\Im \gamma \subseteq S \subseteq X$. Since $h$ is bounded on $S, h \gamma$ is a bounded function and $D(h \gamma)$ is defined.

From now on, let $h$ be bounded on each bounded subset of $\mathcal{X}$.

Definition 3.5. Let $\gamma$ be a rectifiable path in $\mathcal{X}$. We define elevation defect along $\gamma$ as $\Delta(\gamma)=D(h \gamma) \div S(0)$.

Lemma 3.6. The elevation defect yields a weak path valuation.

Proof. Clearly, for a trivial path $\gamma$ we have minimal defect $D(\gamma)=S(0)$, thus, $\Delta(\gamma)=0$. Since the definition of the $c$-cohesiveness considers always neighborhoods with no side-restriction, the defect of a path equals to the defect of its inverse. Moreover, a concatenation $\gamma=\alpha \beta$ has a defect equal to the maximum of $D(\alpha)$ and $D(\beta)$. It remains to show that $\max (D(\alpha), D(\beta)) \div S(0) \leq(\Delta(\alpha) \div$ $S(0))+(\Delta(\beta) \div S(0))$. If $M=\underline{\mathbb{R}}$, then, clearly, $S(0)=0$ and the statement is obvious. Let $M=\underline{\mathbb{N}}$, thus, $S(0)=1$. Let $a=D(\alpha)$ and $b=D(\beta)$. By the definition, $a, b>0$, i.e, $\max (a, b)>1$, thus, we ask whether $\max (a, b)-1 \leq(a-1)+(b-1)$ for some integers $a, b \geq 1$. But $a-1 \geq 0$, thus, if $a \leq b$, then $\max (a, b)=b \leq b+a-1$. For the analogous reason for $b<a$ we have $\max (a, b) \leq a+b-1$, which is equivalent to the required property.

Finally we show that this path valuation enables the description of some concepts from the Definition 3.3.

Theorem 3.7. Let $(\mathcal{X}, h)$ be a topographic space over a self-consistent monoid with the elevation function bounded on every bounded subset. Let $x, y \in X$. Then

(1) $\lambda_{\Delta}(x, y)=0$ iff $x$ and $y$ are mutually nearly accessible,

(2) $\omega_{\Delta}(x, y)=0$ iff $x$ and $y$ are mutually accessible. 
Proof. Clearly, we have

$$
\begin{array}{rlc}
\lambda_{\Delta}(x, y)=0 & \Leftrightarrow & \inf \{D(\gamma) \mid \gamma: x \rightarrow y\}=S(0) \\
& \Leftrightarrow & (\forall c>S(0))(\exists \gamma: x \rightarrow y) D(\gamma)<c \\
\omega_{\Delta}(x, y)=0 & \Leftrightarrow & \bigwedge\{D(\gamma) \div S(0) \mid \gamma: x-\rightarrow y\}=\uparrow 0 \\
& \Leftrightarrow & \bigwedge\{D(\gamma) \mid \gamma: x \rightarrow y\}=\uparrow S(0) \\
& \Leftrightarrow & (\exists \gamma: x \rightarrow y) D(\gamma)=S(0)
\end{array}
$$

Thus, we obtain the mutual (near) accessibility.

\section{TOPOGRAPHIC CHARACTERISTICS}

On every topographic space $\mathcal{X}=(X, M, \delta, h)$, the elevation function yields a pseudogem $\epsilon$ (an elevation distance) given by $\epsilon(x, y)=\mu(h(x), h(y))$ where $\mu$ is the gemF on $M$. Moreover, due to Proposition 2.2 there is an induced gem $(\delta, \epsilon)$ : $X^{2} \rightarrow M^{2}$. There are many more other distance-like characteristics but in order to introduce them we need to study characteristics of paths in topographic spaces. Again, we work with a gem space $\mathcal{X}=(X, M, \delta)$ over a self-consistent monoid.

\subsection{Path characteristics}

Given a path $\gamma: x \rightarrow y$ in a $\mathcal{X}$, one often needs a characteristic which corresponds to elevation fluctuation along a path. These characteristics are widespread namely in context of evaluating the route on the earth's surface. They are rigorously defined in [8], where one can find, besides the elevation distance, concepts of elevation gain and prominence. We base definitions of two groups of characteristics on these concepts for the topographic spaces.

Elevation gain. This group of concepts includes the total amount of ascent $E^{\uparrow}$ and of descent $E^{\downarrow}$ and both together $E^{\uparrow \downarrow}$. Clearly, we should have $E^{\uparrow \downarrow}=E^{\downarrow}+E^{\uparrow}$, and $E^{\downarrow} \div E^{\uparrow}=\epsilon(x, y)$. We define them as follows:

Definition 4.1. For a path $\gamma$, we define

- $E^{\uparrow \downarrow}(\gamma)=l_{\epsilon}(\gamma)$, total elevation movement,

- $E^{\uparrow}(\gamma)=\frac{1}{2}\left(E^{\uparrow \downarrow}(\gamma) \div \epsilon(x, y)\right)$, elevation gain

- $E^{\downarrow}(\gamma)=E^{\uparrow \downarrow}(\gamma) \div E^{\uparrow}(\gamma)$, elevation loss.

Remark 4.2. Since $M$ is either $\underline{\mathbb{R}}$ (which is divisible) or $\underline{\mathbb{N}}$, the value $E^{\uparrow \downarrow}(\gamma) \div$ $\epsilon(x, y)=l_{\epsilon}(\gamma) \div \mu\left(\gamma(0), \gamma\left(m_{\gamma}\right)\right)$ is, by Lemma 2.24 , divisible by 2 , thus, its half is uniquely determined.

Remark 4.3. Each path $\gamma$ in the topographic space $(\mathcal{X}, h)$ yields increasing, constant and decreasing subsets of $M$, i.e., subsets where the mapping $h \circ \gamma$ has the given property. The elevation gain along the path $\gamma$ is equal to the elevation gain on the restriction of $\gamma$ on the increasing subsets only. The total elevation movement is then sum of elevation gain on the increasing subsets and of elevation loss on the decreasing subsets. 
Example 4.4. (1) The assumption of self-consistence is essential: consider e.g., the space $(\underline{\mathbb{Q}}, \mathbb{Q}, \mu)$ with the elevation function

$$
h(x)=\left\{\begin{array}{cc}
x, & x<\sqrt{2} \\
1+x, & \sqrt{2}<x<2 \\
3, & x \geq 2 .
\end{array}\right.
$$

Let $\gamma: 0 \rightarrow 2$ be the (truncated) identity function, then

$$
\begin{aligned}
E^{\uparrow \downarrow}(\gamma)= & \lim _{x \rightarrow \sqrt{2}_{-}}(x-0)+\lim _{x \rightarrow \sqrt{2}_{+}}(3-(1+x)) \\
= & \sqrt{2}+3-1-\sqrt{2}=2,
\end{aligned}
$$

but $\epsilon(\gamma(0), \gamma(m(\gamma)))=\epsilon(0,2)=3-0=3$. Thus, the total elevation movement is smaller than the elevation distance, which makes elevation gain $2 \div 3=1$. That makes no sense to the actual path (in fact, it is the size of the gap instead).

(2) The elevation characteristics may be undefined even when the path is passable. Consider $X=\underline{\mathbb{R}}$ and let $h: \underline{\mathbb{R}} \rightarrow \underline{\mathbb{R}}$ be defined by

$$
h(x)=\left\{\begin{array}{crr}
x\left(1+\cos \left(\frac{2 \pi}{x}\right)\right) & \text { if } & x \neq 0 \\
0 & x=0
\end{array}\right.
$$

Let $\gamma: 0 \rightarrow 1$ be the identity function on $[0,1]$. Then increasing subsets are exactly the intervals $\left[\frac{2}{2 n+1}, \frac{1}{n}\right]$ for every $n \in \mathbb{N}$. The values in their endpoints are always 0 and $\frac{2}{n}$. Thus, $E^{\uparrow}(\gamma)=\sum_{n=1}^{\infty} \frac{2}{n}$, which is infinite.

Prominence characteristics. Another way to characterize the connectivity between the two points in the topographic space is via prominence characteristics. These functions make it possible to calculate distance, similarity or even a significance of a single point within the space. We derive the concepts from the prominence [8], which is one of the measures of a significance of a local maxima.

Definition 4.5. Given a pair of points $x, y$ and a path $\gamma: x \rightarrow y$, we define

$$
\begin{gathered}
\sigma^{+}(\gamma)=\sup \{h(\gamma(t)) \mid t \in M\}, \sigma^{-}(\gamma)=\inf \{h(\gamma(t)) \mid t \in M\}, \\
\underline{\theta}(\gamma)=\mu\left(\sigma^{+}(\gamma), \sigma^{-}(\gamma)\right) .
\end{gathered}
$$

Observe that if the path has the greatest and the least values, then they are equal to $\sigma^{+}(\gamma)$ and $\sigma^{-}(\gamma)$, respectively. The value $\underline{\theta}$ is their symmetric distance.

\subsection{Distances and point characteristics}

In this subsection we use the defined generalized distances for characterization of the properties of a single point. As we will see, some of them make it possible to recognize whether the elevation has an extreme in the given point.

Pseudogems from paths. Once we have introduced path characteristics, we may use the tools of inner gems and its abstraction to obtain new pseudogems. In order to make it possible, we should check their applicability.

Lemma 4.6. The characteristics $E^{\uparrow \downarrow}$ and $\underline{\theta}$ are weak path-valuations. 
Proof. Elevation characteristics: clearly, $E^{\uparrow \downarrow}$ is self-uniform, i.e., $E^{\uparrow \downarrow}(\gamma)=0$ for a trivial path, but it does not imply $\gamma$ is trivial, since this holds for any horizontal path. However, $E^{\uparrow \downarrow}$ is direction independent and additive. Thus $E^{\uparrow \downarrow}$ is a weak path-valuation.

Prominence characteristics: $\underline{\theta}(\gamma)=0$ iff $\gamma$ is horizontal (which includes the trivial paths). Thus, $\underline{\theta}$ is self-uniform and clearly, the value is direction independent. We will show that $\underline{\theta}$ is also sub-additive. Clearly, $\underline{\theta}(\gamma)=\sigma^{+}(\gamma)-\sigma^{-}(\gamma)$. Let $\gamma$ be a concatenation of $\alpha$ and $\beta$. Then we have $\underline{\theta}(\gamma)=\sup _{t} \gamma(t)-\inf _{t} \gamma(t)=$ $\sup \{\sup \alpha(t)-\inf \alpha(t), \sup \beta(t)-\inf \alpha(t), \sup \alpha(t)-\inf \beta(t), \sup \beta(t)-\inf \beta(t)\} \leq$ $\sup \alpha(t)+\sup \beta(t)-\inf \alpha(t)-\inf \beta(t)=\underline{\theta}(\alpha)+\underline{\theta}(\beta)$.

Remark 4.7. No other of the characteristics defined above are weak pathvaluations. Neither of $\sigma^{+}, \sigma^{-}$are self-uniform since trivial path on $x$ has the value $h(x)$, i.e., generally differen from 0 . Moreover $E^{\uparrow}, E^{\downarrow}$ are direction dependent since they count values of mutual inverses.

Consider the space $(X, M, \rho)$ with weak path-valuations $E^{\uparrow \downarrow}$ and $\underline{\theta}$. The Theorem 2.30 induces new pseudogems $E=\lambda_{E^{\uparrow \downarrow}}$ (total elevation distance) and $\theta=\lambda_{\underline{\theta}}$ (prominence distance) given by

$$
\begin{gathered}
E(x, y)=\inf \left\{E^{\uparrow \downarrow}(\gamma) \mid \gamma: x \rightarrow \rightarrow_{\rho} y\right\}, \\
\theta(x, y)=\inf \{\underline{\theta}(\gamma) \mid \gamma: x \rightarrow \rho g\} .
\end{gathered}
$$

Since clearly, $E^{\uparrow \downarrow}(\gamma) \geq \underline{\theta}(\gamma)$ for every $\gamma$ we have $E \geq \theta$.

Point characteristics and extreme detection. Besides these distances, we can derive also characteristics for a single point. Here, we use the main idea that each point $x$ induces a set $Y^{\prime}(x)=h^{-1}(\uparrow h(x)) \backslash\{x\}$. Then the Definition 2.36 gives us assignments $\widetilde{\delta}\left(x, Y^{\prime}(x)\right)$ for a given $\delta$

Let $\delta=\theta$. Then we obtain

$$
\begin{aligned}
\widetilde{\theta}\left(x, Y^{\prime}(x)\right) & =\inf \left\{\theta(x, y) \mid y \in Y^{\prime}(x)\right\} \\
& =\inf _{y \neq x, h(y) \geq h(x) \gamma: x-x_{\rho} y} \underline{\theta}(\gamma) \\
& =\inf _{y \neq x, h(y) \geq h(x) \gamma: \inf _{\infty} y} \mu\left(\sigma^{+}(\gamma), \sigma^{-}(\gamma)\right)
\end{aligned}
$$

Let us explore more the properties of $y$ and $\gamma$ considered in the calculation above. Since $h(x) \leq h(y)$ and we work with

$$
\inf _{\gamma: x-\rightarrow \rho} \mu\left(\sigma^{+}(\gamma), \sigma^{-}(\gamma)\right)
$$

we can assume that $\sigma^{+}(\gamma)=h(y)$, otherwise on each path $x \rightarrow y$ there would be $z$ with $h(z) \geq h(y)$ thus, $y$ (and the path to it from $x$ ) would be dominated by others. Thus we can assume all paths $x \rightarrow y$ contain, besides the endpoints, only elements of smaller elevation.

We will show that this property can be used to detect local maxima of the elevation function. If $M$ is $\mathbb{R}$, then if there is not a local maximum of $h$ in $x$, then there is no nearest higher point $y$ and the infimum of prominence distances (as well as their elevation distances) is zero. On the other hand, if $M$ is $\underline{\mathbb{N}}$ and there is not a local maximum of $h$ in $x$, then there is an adjacent point $y$ higher than $x$ and their prominence distance equals their elevation distance. 
Assume that there is a local maximum of $h$ in $x$. Then each path $\gamma: x \rightarrow-\rightarrow y$ has to pass over a saddle bellow both $x$ and $y$ and does not climb any higher than to $y$. Thus $\underline{\theta}(\gamma)=h(x)-\sigma^{-}(\gamma)+\epsilon(x, y)$ and $\widetilde{\theta}\left(x, Y^{\prime}(x)\right)=\inf \left\{h(x)-\sigma^{-}(\gamma)+\right.$ $\left.\epsilon(x, y) \mid y \in Y^{\prime}(x), \gamma: x \rightarrow y\right\}$. Since $\underline{\theta}(\gamma) \geq \epsilon(x, y)$ in each case, we may subtract $\underline{\theta}(\gamma)-\epsilon(x, y)$. Due to the discussion above, we get always $\underline{\theta}(\gamma)-\epsilon(x, y)=$ $h(x)-\sigma^{-}(\gamma)$. Now we replace $\underline{\theta}$ by $h(x)-\sigma^{-}$in the above expression to get $\left.\inf _{\gamma: x \rightarrow \rho y}\left(h(x)-\sigma^{-}(\gamma)\right)=h(x)-\sup _{\gamma: x \rightarrow x_{\rho} y} \sigma^{-}(\gamma)\right)$. Then we put a new value

$$
L(x)=h(x)-\sup _{y \neq x, h(y) \geq h(x)} \sup _{\gamma: x-\rightarrow \rho y} \sigma^{-}(\gamma) .
$$

The value of $L(x)$ is equal to the elevation distance between $x$ in the local maximum and the most elevated saddle (called key $\mathrm{col}$ ) which separates $x$ from other equally or more elevated point. However, as pointed out in the end of Section 2.4 one has to be careful with the non-emptiness of the corresponding set $Y$. Here, it may happen that the mapping $h$ has its strict global maximum at $x$, i.e., $x=\top$ is a top element of the corresponding order $\leq_{h}$ (it may not exist). Then we purify the definition by:

$$
L(x)=\left\{\begin{array}{cc}
h(x)-\sup _{y \neq x, h(y) \geq h(x) \gamma: x \rightarrow \sup _{\rho} y} \sigma^{-}(\gamma) & x \neq \top \\
h(x) & x=\top .
\end{array}\right.
$$

This function is called lower prominence and we have just proved:

Theorem 4.8. Given a point $x \in X$, then $L(x) \neq 0$ iff $h$ has a local maximum at $x$.

By an analogy, we may define an upper prominence

$$
U(x)=\inf _{y \neq x, h(y) \leq h(x)} \inf _{\gamma: x \rightarrow-\infty} \sigma^{+}(\gamma)-h(x)
$$

which detects local minima in the same manner as above. However, one has to be careful since $U$ is undefined in the strict global minimum (though we may complete the definition by setting the height of the global maxima minus the height of this global minimum.)

Using the total elevation distance we can obtain similar result:

Theorem 4.9. Let $x \in X$ and

$$
R(x)=\left\{\begin{array}{cl}
\inf \{E(x, y)-\epsilon(x, y) \mid h(y) \leq h(x), y \neq x\} & x \neq \top \\
h(x) & x=\top .
\end{array}\right.
$$

Then $R(x) \neq 0$ iff $h$ has a local maximum at $x$.

Proof. Following the same argumentation as above, $E^{\uparrow \downarrow}(\gamma)-\epsilon(x, y)$ converges to zero (for considered $h(y) \geq h(x), y \neq x, \gamma: x \rightarrow-\rightarrow y$ ) iff $x$ is not in the local maximum of $h$. The proof differs by reformulation of the expression; here we have $\inf \left\{E^{\uparrow \downarrow}(\gamma)-\epsilon(x, y) \mid \gamma: x \rightarrow \rightarrow_{\rho} y\right\}=E(x, y)-\epsilon(x, y)$ thus we get the assignment stated in the theorem.

Analogously, we can also construct a function $S: X \rightarrow M$ derived from $E$ which detects local minima. 
Remark 4.10. For each prominence $F: X \rightarrow M$ we obtain a new topographic space $\mathcal{X}_{p}=(X, M, \delta, F)$. The upper prominence of $\mathcal{X}_{L}=(X, M, \delta, L)$ will remain the same since now the strict local maxima will have elevation of their prominence and the rest will have the value zero. Thus $\mathcal{X}_{L}$ can be used for the discretion of the original space, which preserves the upper prominence. Indeed, this process makes it possible to replace a continuous function on a metric space by a function with a finite or countable support (set of points with nonzero value).

\subsection{Abstract topographic characteristics}

As shown in the paragraph on abstract inner gem in the Section 2.4, all of the previous concept has an abstract counterpart, which provides even richer treatment. Namely, for a pseudogem space $(X, M, \delta)$ over any value monoid $M$, the weak path-valuations $E^{\uparrow \downarrow}$ and $\underline{\theta}$ provide, due to Theorem 2.30, new abstract pseudogems $\widetilde{E}=\omega_{E \uparrow \downarrow}$ and $\widetilde{\theta}=\omega_{\underline{\theta}}$. Moreover, one can construct analogy for the functions $L, U, R, S: X \rightarrow M$ but the extreme detection procedure would not work as smooth as in the concrete case. However, we may consider multiple (weak) pathvaluations to construct vectors and use the above procedure to create new, more useful abstract pseudogems. More precisely: if we take path valuations $J_{1}, \ldots, J_{n}$, with $J_{i}$ mapping paths into a monoid $M_{i}$, then we obtain

$$
\omega_{J_{1}, \ldots, J_{n}}(x, y)=\bigwedge_{\gamma: x-\rightarrow y}\left(J_{1}(\gamma), \ldots, J_{n}(\gamma)\right) \in \mathcal{W}\left(N_{1} \times \cdots \times N_{n}\right) .
$$

Recall that, in general, the dissimilarities can be summed, maximized or collected into a multi-component vector. Moreover, as noted in Remark 1, each pseudogem gives rise to a weak path-valuation. Thus we may obtain pseudogems such as:

$$
\omega_{l, E^{\uparrow \downarrow}}, \quad \omega_{\delta, \theta}, \quad\left(\omega_{l, \epsilon}, \lambda_{\theta}\right), \quad\left(\delta, E, \omega_{E, \theta}\right), \quad\left(\lambda_{\epsilon}, \delta+\epsilon, \Delta\right), \quad \ldots
$$

These distances capture more information than a single value, thus we can describe the space with such distances. Some abstract pseudogems will be evaluated in the example at Figure 3.

\subsection{Prominence connectedness}

We will now focus on the connectedness w.r.t. prominence distance.

Observation 4.11. Consider a gem space $\mathcal{X}=(X, M, \delta)$ over a self-consistent monoid and a topographic space $(\mathcal{X}, h)$. One can easily verify the following:

$$
\theta(x, y)=b \Leftrightarrow(\forall \gamma: x \rightarrow y) b \leq \underline{\theta}(\gamma) \wedge(\forall a>0)(\exists \gamma: x \rightarrow y) \underline{\theta}(\gamma)<a+b .
$$

In the case of $M=\underline{\mathbb{N}}$, we get even simpler formula:

$$
\theta(x, y) \leq b \Leftrightarrow(\exists \gamma: x \rightarrow-\rightarrow y) \underline{\theta}(\gamma) \leq b .
$$

Namely, $\theta(x, y) \leq 1 \Leftrightarrow(\exists \gamma: x \rightarrow y) \mu\left(\sigma^{+}(\gamma), \sigma^{-}(\gamma)\right) \leq 1$ which yields the existence of a passable path $\gamma: x \rightarrow y$.

Due to Proposition 2.2, each topographic space can be factorized over the equivalence relation $\sim_{\theta}$. Since $\mu(k, l)=k \div l$ we have $x \sim_{\theta} y \Leftrightarrow(\forall a>0)(\exists \gamma: x \rightarrow-$ $y)\left(\sigma^{+}(\gamma) \div \sigma^{-}(\gamma)\right)<a$. That means that: 
Lemma 4.12. The equivalence $\sim_{\theta}$ decomposes $X$ into maximal nearly horizontal subspaces.

We obtain a new topographic space $\mathcal{X}_{\theta}=(X, M, \theta, h)$.

Definition 4.13. A point $x \in X$ is called a pole point of the topographic space $(X, M, \delta, h)$ if there is no nontrivial passable path starting from it.

Lemma 4.14. Let $\mathcal{X}$ be a dense space with no pole points. Then $\mathcal{X}_{\theta}$ has no isolated points.

Proof. Let $x \in X$ and $e>0, O_{\mu}^{*}(0, e)$. Then there exists $y \in X$ and a passable path $\gamma: x \rightarrow y$. If $M$ has an atom, then $e>1$ and for $u=\gamma(1) \mu(h(x), h(u))=$ $\mu(h \gamma(0), h \gamma(1)) \leq 1$ thus for $\beta=\gamma_{0,1}: x \rightarrow u$ there is $\underline{\theta}(\beta) \leq 1<e$. Now let $M$ have no atom. Then there exists $r=\frac{e}{3} \in M$ such that $0<2 r<e$. Then $O_{\delta}^{*}(x, r) \neq \emptyset$ since $X$ is dense. Since $x$ is not a pole point, there exists a passable path $\alpha$ from $x$, thus $h \circ \alpha$ is cohesive. Therefore, there exists $d \in M$ such that $O_{\mu}^{*}(0, d) \neq \emptyset$ and for every $0<c<d h(\alpha(c)) \in O_{\delta}(x, r)$. Therefore, $\mu(h(x), h(\alpha(c)))<r$, thus for every $b, c<d, \mu(h(\alpha(b)), h(\alpha(c)))=h(\alpha(b)) \div$ $h(\alpha(c)) \leq(h(\alpha(b)) \div h(u))+(h(\alpha(c)) \div h(u))<r+r<e$.

It can be helpful for a description of near passability as shown in the following theorem:

Theorem 4.15. Let $\mathcal{X}$ be a dense space with no pole points. Then if the space $\mathcal{X}_{\theta}$ is path-connected, $\mathcal{X}$ is nearly passable.

Proof. Let $\mathcal{X}_{\theta}$ be path-connected and $x, y \in X$ be two distinct points. Then there exists a path $\gamma: x \rightarrow y$ with $m=m(\gamma)$ which is cohesive w.r.t. the pseudogem $\theta$. Thus, for each $t \in M$ and $e>0$ satisfying $\emptyset \neq O_{\theta}^{*}(\gamma(t), e$ ) (which is satisfied for every $e>S(0)$ and $t \in M$ due to the previous lemma), there exists $d=d_{t, e}$ such that $\emptyset \neq O_{\mu}^{*}(t, d)$ and, for each $s \in O_{\mu}^{*}(t, d), \theta(\gamma(t), \gamma(s))<e$.

Our goal is to find, for every $c>S(0)$, a path $\alpha: x \rightarrow y$ such that $h \circ \alpha$ is $c$-cohesive. We will find this path as a certain concatenation of paths but its construction will be made separately for atomic and non-atomic case.

(1) Let $M$ have an atom, i.e., $M \cong \mathbb{N}$. Let $t \in M$ and $e=2$, then by cohesiveness (w.r.t. $\theta$ ) of $\gamma$ we have $d=d_{t, 2}$ satisfying the condition in $D R_{1}(\gamma, t, 2, \gamma(t))$. Therefore, $\theta(\gamma(t), \gamma(t+1))<2$, i.e., by the last part of Observation 4.11 there exists a passable path $\beta_{t}: \gamma(t), \gamma(t+1)$. Since concatenation of passable paths is, clearly, passable, we get a passable path

$$
\alpha=\beta_{0} \beta_{1} \ldots \beta_{m-1}: x \rightarrow y .
$$

Thus the space is passable, thus nearly passable.

(2) Suppose $M$ has no atoms, i.e., $M \cong \mathbb{R}$. Let $e=\frac{c}{3}$. Since, for each $t, \gamma(t)$ is not isolated by the previous lemma $\emptyset \neq O_{\theta}^{*}(\gamma(t), e)$. We construct two sequences of scalars $p_{i}$ (parameters) and $r_{i}$ (radii) such that $\theta\left(\gamma\left(p_{i}\right), \gamma\left(p_{i+1}\right)\right)<e$. Consider the sets $R_{t, e}$ from Lemma 2.13.

If, for some $t$, the set $R_{t, e}$ is not bounded, it means that the whole set $\underline{\mathbb{R}}$ is the neighborhood $N$ of $t$ such that $\gamma(N) \subseteq O_{\theta}(u, e)$ (here $u=\gamma(t)$ ), 
thus $x, y \in O_{\theta}(u, e)$, i.e., $\theta(x, u)<e, \theta(u, y)<e$, thus we get a three element sequence $(x, u, y)$ satisfying the required condition.

Suppose $R_{t, e}$ is bounded for each $t \in \mathbb{R}$. Then, by Lemma 2.13 we can take $d_{t, e}=\sup R_{t, e}$. The notation will be simplified to $d_{t, e}=d_{t}$.

Let $p_{0}=x, r_{i}=\frac{d_{p_{i}}}{2}, p_{i+1}=p_{i}+r_{i}$ for $i \in \underline{\mathbb{N}}$. Then, for every $i$, $p_{i+1} \in B_{\mu}^{*}\left(p_{i}, r_{i}\right) \subset O_{\mu}^{*}\left(p_{i}, d_{p_{i}}\right)$, therefore $\theta\left(\gamma\left(p_{i}\right), \gamma\left(p_{i+1}\right)\right)<e$.

We will show that there exists $n$ such that $p_{n} \geq m$. Clearly, $\left(p_{i}\right)_{i}$ is an infinite increasing sequence. If there is no such index $n$, then, for every $i \in \underline{\mathbb{N}}, p_{i}<m$. Then $\left(p_{i}\right)_{i}$ is increasing and bounded, thus it has a limit $L \leq m$. We will show that such situation can occur only if $L=p_{j}$ for $j \geq n$ for some $n$. Assume that it is not true, i.e., the sequence is a strictly increasing, thus $\left(p_{i+1}-p_{i}\right)_{i}$ is decreasing, positive and convergent to 0 .

Since $\gamma$ is cohesive w.r.t. $\theta$, and since $O_{\theta}^{*}\left(\gamma(L), \frac{e}{2}\right) \neq \emptyset$ (each point of $X$ is accessible), then $\exists g \in \underline{\mathbb{R}}$ such that $O_{\mu}^{*}(L, g) \neq \emptyset$ (i.e., $g>0$ ) such that $\gamma\left(O_{\mu}^{*}(L, g)\right) \subseteq O_{\theta}\left(\gamma(L), \frac{e}{2}\right)$. Let $k \in(L-g, L)$, then $O_{\mu}^{*}(k, g-$ $(L-k)) \subseteq O_{\mu}^{*}(L, g)$ and for every $k^{\prime} \in O_{\mu}^{*}(L, g)$ we have $\theta\left(\gamma(k), \gamma\left(k^{\prime}\right)\right) \leq$ $\theta(\gamma(k), \gamma(L))+\theta\left(\gamma(L), \gamma\left(k^{\prime}\right)\right)<\frac{e}{2}+\frac{e}{2}=e$. Thus $\gamma\left(k^{\prime}\right) \in O_{\theta}(\gamma(k), e)$. Therefore, $g-(L-k) \in R_{k, e}$, i.e., $g-(L-k) \leq d_{k}$.

Since $L=\lim _{i \in \mathbb{N}} p_{i}$ and, by assumption, $p_{i+1}-p_{i}>0$ for every $i$, there exists $j \in \underline{\mathbb{N}}$ such that $\mu\left(L, p_{j}\right)<\frac{g}{3}<g$, i.e., $3\left(L-p_{j}\right)<g$. Moreover, $p_{j}<p_{j+1}<L$, thus $L-p_{j}>p_{j+1}-p_{j}=r_{j}=\frac{d_{p_{j}}}{2}$. Since $p_{j}$ satisfies the properties of $k$ above, we have $g-L+p_{j} \leq d_{p_{j}}$. Altogether we have $L-p_{j}>\frac{d_{p_{j}}}{2} \geq \frac{g-L+p_{j}}{2}$, thus $2\left(L-p_{j}\right)>g-L+p_{j}$, i.e., $3\left(L-p_{j}\right)>g$, which contradicts $3\left(L-p_{j}\right)<g$. Therefore, the sequence of $p_{i}$ converges by stopping (at $m$ ) after a finite number $n$ of steps.

Since $n$ is finite, there exists $d=\frac{1}{2} \inf \left\{d_{p_{i}} \mid i \in\{0, \ldots, n\}\right\}>0$. Let $k \in\{0, \ldots, n\}, x_{k}=\gamma\left(p_{k}\right)$ and $x_{k+1}=\gamma\left(p_{k+1}\right)$. Then $\theta\left(x_{k}, x_{k+1}\right)<e$, i.e. by Observation 4.11, $\left(\exists b_{k}<e\right)\left(\forall \beta: x_{k} \rightarrow x_{k+1}\right) b_{k} \leq \underline{\theta}(\beta) \wedge(\forall a>$ $0)\left(\exists \beta_{a, k}: x_{k} \rightarrow x_{k+1}\right)\left(\sigma^{+}\left(\beta_{a, k}\right)-\sigma^{-}\left(\beta_{a, k}\right)\right)<a+b_{k}$.

Let $b=\max \left\{b_{k} \mid k \in\{0, \ldots, n\}\right\}, a=e-b$ and

$$
\alpha=\beta_{a, 0} \beta_{a, 1} \ldots \beta_{a, n-1}
$$

which is a concatenation of a finite number of paths. We will show that this $\alpha$ satisfies the required condition. Let $t \in M, t \leq m$, then there exists $k=k(t) \in \underline{\mathbb{N}}$ such that $p_{k} \leq t<p_{k+1}$. Let $s \in O_{\mu}^{*}(t, d)$, then $s \in\left[p_{k-1}, p_{k+2}\right)$. Assume that $s<t$. Then $k(s)$ equals $k-1$ or $k$. Thus $\mu(h(\alpha(s)), h(\alpha(t)))=h(\alpha(s)) \div h(\alpha(t)) \leq\left(\sigma^{+}\left(\beta_{a, k-1}\right)-\sigma^{-}\left(\beta_{a, k-1}\right)\right)+$ $\left(\sigma^{+}\left(\beta_{a, k}\right)-\sigma^{-}\left(\beta_{a, k}\right)\right) \leq a+b_{k-1}+a+b_{k} \leq 2 a+2 b=2 e=\frac{2}{3} c<c$. Analogously we prove $\mu(h(\alpha(s)), h(\alpha(t)))<c$ for $s>t$.

Therefore, we have a path $\alpha=\alpha_{c}$ such that, for every $g>c$ and for every $t \in M$, there exists $d>0$ (chosen above) such that $\emptyset \neq O_{\mu}^{*}\left(p_{k}, d\right)$ (clearly, since $M=\underline{\mathbb{R}}$ ) and for every $s \in O_{\mu}^{*}(t, d)$ we have $t \div s<d$, which implies, as shown above, $h(\alpha(s)) \in O_{\mu}(h(\alpha(t)), c) \subset O_{\mu}(h(\alpha(t)), g)$. Thus $h \circ \alpha$ is $c$-cohesive. 
The following property gives another implication.

Lemma 4.16. Let $\mathcal{X}$ have no isolated points. Then every passable path in $(\mathcal{X}, h)$ is a path in $\mathcal{X}_{\theta}$.

Proof. Let $\gamma: x \rightarrow y$ be a passable path in $(\mathcal{X}, h)$. We will show that it is a path w.r.t. the pseudogem $\theta$. Let $t, e \in M, u=\gamma(t)$, then if $O_{\theta}^{*}(u, e) \neq \emptyset$ we look for $d \in M$ such that $\emptyset \neq \gamma\left(O_{\mu}^{*}(t, d)\right) \subseteq O_{\theta}^{*}(u, e)$.

Let $M$ have no atoms. Since $O_{\theta}^{*}(u, e) \neq \emptyset$ there exists $v$ such that $\theta(u, v)<e$, thus there exists a path $u \rightarrow v$, then since $u$ is not isolated, the neighborhood $O^{*}\left(u, \frac{e}{2}\right)$ cannot be empty. Therefore, there exists $d=d_{t, \frac{e}{2}}$ satisfying the nonemptiness condition and, for every $s \in O^{*}(t, d), \mu(h \gamma(t), h \gamma(s))<\frac{e}{2}$. Suppose $s<$ $t$. Thus the subpath $\gamma_{s, t}: \gamma(s) \rightarrow \gamma(t)$ leads through points $\gamma(r), r \in[s, t]$ each of which satisfies $\mu(h \gamma(t), h \gamma(r))<\frac{e}{2}$. Then $\underline{\theta}\left(\gamma_{s, t}\right)<\left(h \gamma(t)+\frac{e}{2}\right)-\left(h \gamma(t)-\frac{e}{2}\right)=e$. Since $\theta(s, t) \leq \underline{\theta}\left(\gamma_{s, t}\right)$, we get the statement.

If $M$ is atomic, e.g., $M=\underline{\mathbb{N}}$ then by the argumentation from the proof of the previous theorem, all we need to show is that, for every $t \in \underline{\mathbb{N}}, \theta(\gamma(t), \gamma(t+1)) \leq 1$. But that is, due to the same discussion, equivalent to the existence of a passable path $\gamma(t) \rightarrow \gamma(t+1)$, which follows from our assumption.

Example 4.17. The converse of the above implications is not true. Figure 1 shows two counterexamples. The shading determines the value of the elevation, which is also shown intuitively as a height. Both spaces consist of two subsets. The right semiopen rectangle (appearing as a parallelogram), which does not contain the rear left side, is horizontal with a constant elevation $p$. The left semiopen rectangle (does not contain the rear right side) has increasing value of elevation from the rear (the value strictly above $p$ ) right to left front edge. However, the rear right edge does not belong to the rectangle and makes up the third part, it is again horizontal subspace with a value $q>p$. The spaces differ by an assignment in a single point $z$. In the case of a), $z$ is not a point of the space, while $z$ is included in the space $\mathrm{b}$ ) and $h(z)=p$. This difference causes a major difference between the spaces. The space in a) is nearly passable but not $\theta$-connected. For each pair of points, e.g., $u, v$, and $c>0$ there exists a path $\gamma$ such that $h \circ \gamma$ is $c$-cohesive. That means that, for every $e>c$, the discontinuity step is smaller than $e$. Here, we show such a path. The space is not $\theta$-connected, since the points $u, v$ cannot be connected by a path w.r.t. $\theta$. Such a path would have to map $\underline{\mathbb{R}}$ into both rectangular parts of the space, thus for each element $t$ and a chosen threshold $c$ there must exist $d$ such that for each element $s$ from its $d$-neighborhood $\theta(\gamma(s), \gamma(t))<c$. That means that there exists a path $\beta: \gamma(t) \rightarrow \gamma(s)$ with values of $\sigma^{+}(\beta)-\sigma^{-}(\beta)$ close to $c$ (in a sense of Observation 4.11). Let $t$ be in the right part. Then $h \gamma(t)=p$ and $h \gamma(s)>p$. Thus for $e<h \gamma(s)-p$ we have $\sigma^{+}(\beta)-\sigma^{-}(\beta) \geq h \gamma(s)-p>e$, which is a contradiction.

The space on $\mathrm{b}$ ) is $\theta$-connected. Each pair of points, e.g., $u, v$ can be connected by a path w.r.t. the pseudogem $\theta$. Such paths are piecewise cohesive paths with gaps separated only by nearly horizontal subset. Namely, if the path leads from $u$ towards $y$ (which does not belong to the space) and then jumps to $z$ and continues to $v$, we have the required property. Indeed, for every $e>0$, we can find a radius $d>0$ such that any parameter $s$ in the $\mu$-distance from $t$ smaller, then $d$ has 
$\theta(\gamma(s), \gamma(t))<e$. This can be seen on the picture where a path $\beta$ (dashed line) from $x=\gamma(s)$ to $y=\gamma(t)$ with the $\underline{\theta}(\beta)=c$ which can be made arbitrarily small. On the other hand, this topographic space is, clearly, not passable since there is no passable path from $u \rightarrow v$ (each path has to overcome the step of nonzero height).

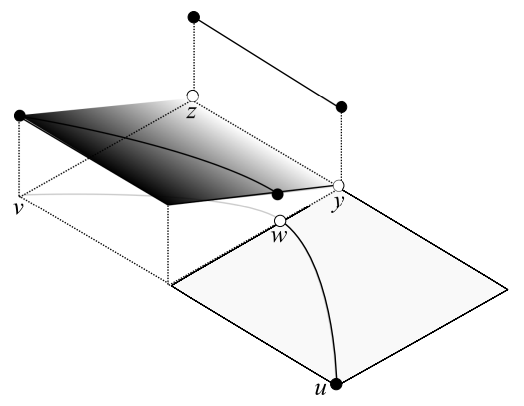

a)

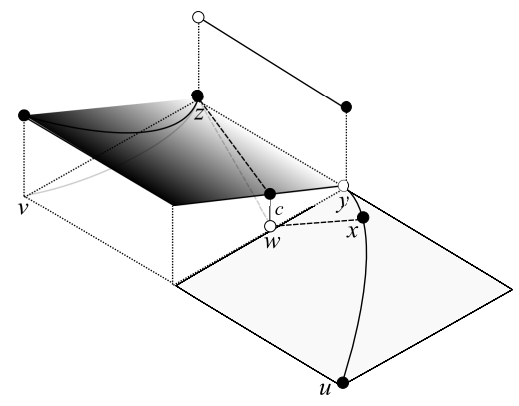

b)

Figure 1. Sketches of topographic spaces over $\underline{\mathbb{R}}$. a) Nearly passable space which is not $\theta$ connected. b) $\theta$-connected space which is not passable.

Corollary 4.18. The above theorem and example provide us with the properties of dense topographic spaces without pole points:

$$
\text { passable } \Rightarrow \theta \text {-connected } \Rightarrow \text { nearly passable }
$$

with neither of the implications being reversible.

One can substitute $E$ for $\theta$ in the above procedure and ask about the validity. Since $E>\theta$, we get, under the assumptions of space denseness that $E$ connectedness implies $\theta$-connectedness. It can be a task for next research to study relations between the topographic characteristics and their abstractions.

\section{Applications}

The theory of topographic spaces, as presented here, may have various applications in mathematics and in other sciences, namely in geography, both physical and socioeconomic, as well as in the digital geometry, a theory beyond the image processing. We will sketch the main line of theoretical application covering more appearances of topographic spaces. As noted in the introduction of the previous section, one of the main theories dealing with topographic spaces is that of digital terrain modeling.

Suppose there is an original topographic space $\mathcal{X}$ over $\underline{\mathbb{R}}$, thus a metric space with an elevation function. The space does not have to be Euclidean, since Earth is not a plane and it can be approximated by a sphere or an ellipsoid. The space is then scanned and transformed into a digital representation. If it is a raster image, then we have the data in a digital space. Other possible presentations are by 
contours (isohypses) and shading, which can be seen as topographic space over $\mathcal{X}$ with elevation of nonzero defect, indeed, the defect is equal to the vertical resolution, i.e., the vertical distance between two consecutive isohypses.

Particularly Theorem 4.15 can be helpful for the detection of near passability of the space. The near passable spaces are of a special interest. Indeed, in the real world practice, problems of accessibility are very topical. Consider, e.g., a project of a university campus and its wheel-chair accessibility. Clearly, if the we model the campus as a topographic space, with height of the ground and halls (on a given floor) being the elevation function (generally not cohesive since the structures usually involve vertical steps- walls, stairs, fences, etc), then its passability would certainly guarantee accessibility of each point from any other. However, the passability is not needed because the precise cohesiveness is not necessary in a real world. In fact, we can be satisfied with some $c$-cohesiveness of a path since each means of transport (e.g., a car, wheel-chair, bike, pedestrians feet or roller-blades), has its own vertical limit of passability (a height of a possible step for a pedestrian, an unevenness of a surface for a wheel-chair or a roller-blade, etc.). Thus, a near passable space is such where each "real" means of transport enables the accessibility of any point. The Theorem 4.15 yields a sufficient condition which can be syntactically verified by tools of connectedness in a metric space or a graph.

On the other hand, considering the wheel-chair user, the level of unevenness is not the only criterion of passability, since the ride on a wheel-chair has also its slope limits. Such a problem would be more tricky to describe in the real setting, but becomes much simpler when transferred into the discrete space. The space can be rasterized with a required level of (in)accuracy so that the unattainable slope (expressed as some ratio of change of elevation with a change of a position) will become as a large vertical step in the newly acquired digital space. Then again, the passable/ $\theta$-connected / nearly passable paths will be of our interest.

To conclude, we show some examples of the topographic characteristics on gray level and raster images in Figure 2. The raster image was obtained from the gray level real image by most abundant value (the mode) on the equivalence class via the mapping described in the Example $2.16(5)$. While the original space is $\left(\mathbb{R}^{2}, \underline{\mathbb{R}}, \mathrm{e}, h\right)$, the raster image is a topographic space $\left(\mathbb{Z}^{2}, \underline{\mathbb{N}}, \mathrm{w}, \bmod _{h}\right)$.

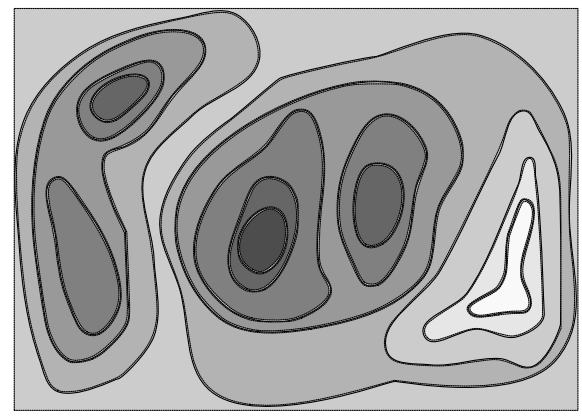

\begin{tabular}{|l|l||l||c|c|c|c|c|c|c|}
\hline 2 & 2 & 4 & 3 & 2 & 2 & 2 & 3 & 2 & 2 \\
\hline 2 & 6 & 4 & 3 & 2 & 4 & 4 & 3 & 3 & 3 \\
\hline 4 & 4 & 3 & 4 & 5 & 4 & 5 & 4 & 2 & 2 \\
\hline 4 & 4 & 2 & 5 & 6 & 5 & 6 & 4 & 2 & 2 \\
\hline 4 & 3 & 3 & 5 & 7 & 4 & 5 & 2 & 0 & 2 \\
\hline 4 & 3 & 3 & 3 & 5 & 4 & 3 & 2 & 1 & 2 \\
\hline 2 & 3 & 2 & 3 & 3 & 3 & 3 & 3 & 3 & 2 \\
\hline
\end{tabular}

Figure 2. Representation of the topographic space by isohypses and grayness levels and by raster image. 
The paths connecting points are shown in Figure 3. The corresponding paths in the isohyptic image are depicted. While $b$ is still non-dominated, $a$ is dominated by the path $d$ which would have the same raster counterpart as $b$. The path $c$ on the left is the shortest, thus, non-dominated, while its raster counterpart is dominated by $b$. Moreover, while $a$ and $b$ on the right are passable, the path $c$ is only 2-passable. The total elevation movement along the paths on the left can easily be calculated as a number of crossed isohypses. The space is, clearly, not passable since, e.g., the upper left corner has no passable connection to the rest of the space. On the left image we cannot say anything about the passability since only finite number of isohypses and gray levels is shown. Topographic features
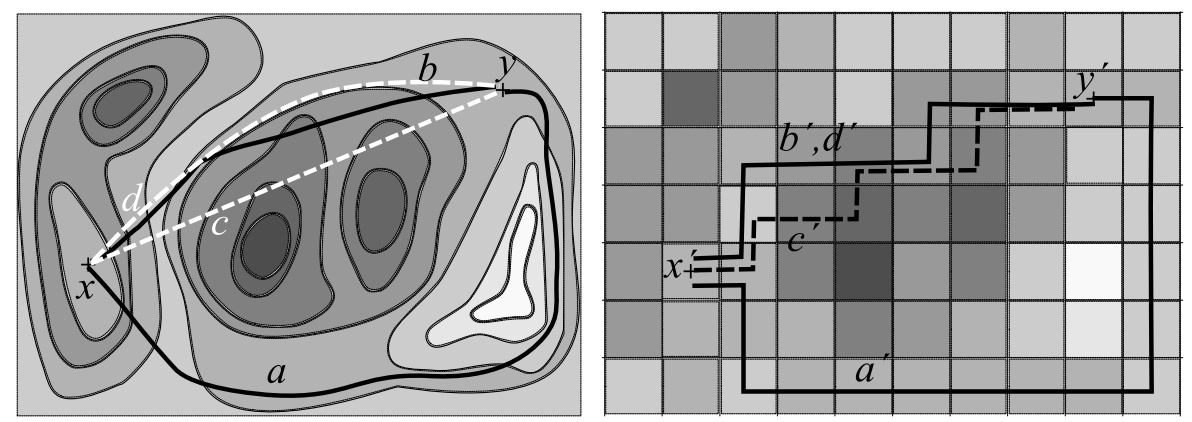

Figure 3. Paths connecting points $x$ and $y$. The paths $a$ and $b$ on the raster image are nondominated and they determine the total topographic distance of the points $\omega_{l, E \uparrow \downarrow}(x, y)=$ $(4,18) \wedge(6,10)$, here the first component is the length and the second is the total elevation movement along the path.

such as local extrema and key saddles are in Figure 4. We can see that some of them are preserved while others are not with some new ones were created.
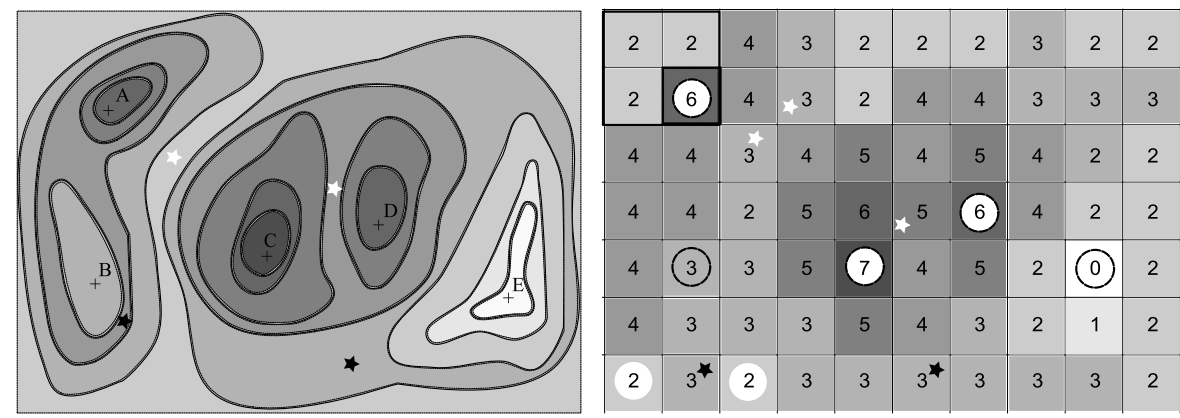

Figure 4. The labeled points are strict local extrema. The edged circles on the right are their positions and full circles are strict local extrema in the raster image. The dark borders denote the regions of passability. The stars denote the key saddles, which make it possible to count the lower prominence. Its values are in the maxima: $L(A)=3, L(C)=7, L(D)=1$. Values of the upper prominence are in the minima: two unlabeled on the left: $U(-)=1$ in both cases, $U(B)=0, U(E)$ is undefined, since $E$ is a global minimum. 
Figure 5 shows balls with respect to the prominence gem with a given center point in two topographic spaces.
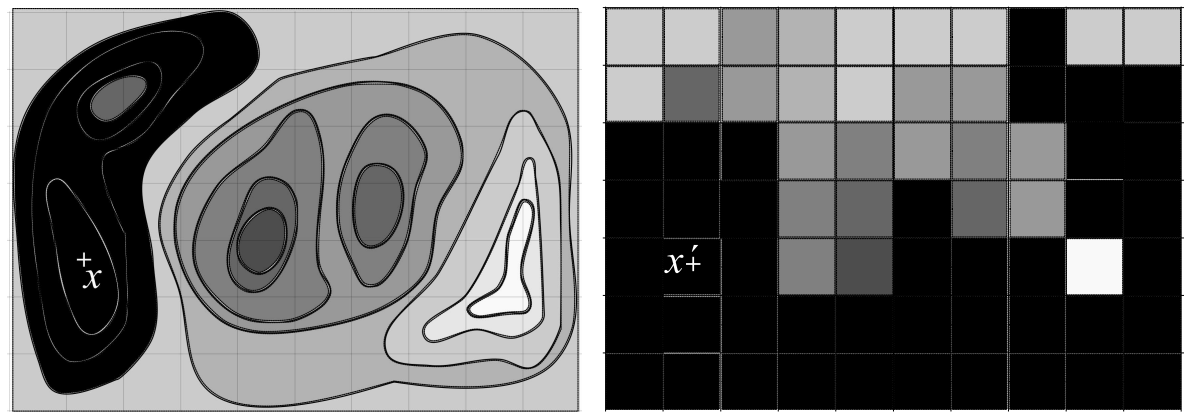

Figure 5. Prominence balls in topographic spaces. In both spaces the labeled point $x, x^{\prime}$ is the center of the ball filled with black. The gem is $\theta$ and radius is 2 in both cases.

\section{REFERENCES}

[1] R. Bělohlávek, Fuzzy Relational Systems: Foundations and Principles, FSR International Series on Systems Science and Engineering 20, Kluwer Academic Publishers, Norwell, MA, USA, 2002.

[2] R. N. Ball and A. Pultr, Quotients and colimits of $\kappa$-quantales, Topol. Appl. 158 (2011), 2294-2306.

[3] J.-P. Barthélemy, F. Brucker and C. Osswald, Combinatorial optimization and hierarchical classifications, Quarterly Journal of the Belgian, French and Italian Operations Research Societies 2 (2004), 179-219.

[4] D. Burago, Y. Burago and S. Ivanov, A Course in Metric Geometry, Graduate Studies in Mathematics 33, AMS, 2001.

[5] P. Bertrand, Set systems and dissimilarities, Eur. J. Combin. 21 (2000), 727-743.

[6] R. Heckmann, Similarity, topology and uniformity, Journal of Logic and Algebraic Programming 79 (2010), 10-31.

[7] G. T. Herman, Geometry of Digital Spaces, Applied and Numerical Harmonic Analysis, Birkhäuser, Basel, 1996.

[8] A. Helman, The Finest PeaksProminence and Other Mountain Measures, Trafford Publishing, 2005.

[9] M. Krötzsch, Generalized ultrametric spaces in quantitative domain theory, Theor. Comput. Sci. 386 (2006), 30-49.

[10] S. Mac Lane, Categories For the Working Mathematician, Springer-Verlag, New York, 1971.

[11] J. Paseka and J. Rosický, Quantales, in: B. Coecke et al. (eds.), Current Research in Operational Quantum Logic, Algebras, Categories, Languages, Workshop, Free Univ. of Brussels, Belgium, June of 1998 and May of 1999, Dordrecht, Kluwer Academic Publishers, Fundam. Theor. Phys. 111 (2000), 245-262.

[12] J. Pavlík, Free algebras in varieties, Arch. Math. 46 (2010), 25-38.

[13] J. Pavlík, Thresholding of a digital image by free terms, J. Math. Imaging Vis. 51 (2015), 338-354.

[14] J. Pavlík, Abstract approach to image segmentation, to appear.

[15] J. Pavlík, Unified approach to graphs and metric spaces, to appear.

[16] R. J. Peckham and G. Jordan (eds.), Digital Terrain Modelling, Lecture Notes in Geoinformation and Cartography, Springer-Verlag, 2007.

[17] F. F. Pan and H. Sheng-Wei, Free Q-algebras, Fuzzy Set. Syst. 247 (2014), 138-150. 
[18] R. Kopperman, All topologies come from generalized metrics, Am. Math. Mon. 95 (1988), 89-97.

[19] K. Rosenthal, Quantales and Their Applications, Pitman Research Notes in Mathematics Series 234, Harlow: Longman Scientific \& Technical, New York, John Wiley \& Sons, 1990.

[20] S. Solovjovs, A representation theorem for quantale algebras, in: Contributions to General Algebra 18, Heyn, Klagenfurt, 2008, 189-197.

[21] W. R. Tunnicliffe, The free completely distributive lattice over a poset, Algebra Univ. 21 (1985), 133-135.

Jan Pavlík, Institute of Mathematics, Faculty of Mechanical Engineering, Brno University of Technology, Technická 2, 61669 Brno, Czech Republic

e-mail: pavlik@fme.vutbr.cz 
